\title{
WCSPH viscosity diffusion processes in vortex flows
}

\author{
F. Macià, J. M. Sánchez, A. Souto-Iglesias and L. M. González*,† \\ Naval Architecture Department (ETSIN), Technical University of Madrid (UPM), 28040 Madrid, Spain
}

\begin{abstract}
SUMMARY
The aim of this paper is to clarify the role played by the most commonly used viscous terms in simulating viscous laminar flows using the weakly compressible approach in the context of smooth particle hydrodynamics (WCSPH). To achieve this, Takeda et al. (Prog. Theor. Phys. 1994; 92(5):939-960), Morris et al. (J. Comput. Phys. 1997; 136:214-226) and Monaghan-Cleary-Gingold's (Appl. Math. Model. 1998; 22(12):981-993; Monthly Notices of the Royal Astronomical Society 2005; 365:199-213) viscous terms will be analysed, discussing their origins, structures and conservation properties. Their performance will be monitored with canonical flows of which related viscosity phenomena are well understood, and in which boundary effects are not relevant. Following the validation process of three previously published examples, two vortex flows of engineering importance have been studied. First, an isolated Lamb-Oseen vortex evolution where viscous effects are dominant and second, a pair of co-rotating vortices in which viscous effects are combined with transport phenomena. The corresponding SPH solutions have been compared to finite-element numerical solutions. The SPH viscosity model's behaviour in modelling the viscosity related effects for these canonical flows is adequate. Copyright (C) 2011 John Wiley \& Sons, Ltd.
\end{abstract}

KEY WORDS: WCSPH; particles; vortex; Lagrangian; viscous; Lamb-Ossen

\section{INTRODUCTION}

The important role that viscosity plays in many engineering and physical phenomena (boundary layers and force evaluations, separation, transition flows, shear flows, etc.) underlines the need for a better understanding of modelling techniques of laminar viscous flows.

Modelling low Reynolds number viscous flows does not present excessive difficulties for industrial-focused computational fluid dynamics (CFD) methods such as finite volume (FVM) or finite element methods (FEM), but these methods encounter difficulties when dealing with problems such as highly distorted free surface flows, where SPH is in general the first option. The SPH method is also successful in modelling the bulk flow of these very turbulent problems. Vila [1] showed the consistency of the SPH approximation for solving the Euler equations but no similar result regarding the Navier-Stokes equations has been obtained so far. Motivated by a series of studies on SPH simulations of free surface viscous [2,3] and vortical flows [4], it became apparent to the authors the need for a better understanding of the SPH modelling of viscosity related phenomena. The weakly compressible formulations (WCSPH) were considered the most adequate SPH models to tackle free surface flows which combine fragmentation and splashing, and they are also a better option for these problems than mesh-based methods, including re-meshed SPH [5]. Consequently, the WCSPH approach to laminar flows will be used in this paper.

${ }^{*}$ Correspondence to: L. M. González, Naval Architecture Department (ETSIN), Technical University of Madrid (UPM), 28040 Madrid, Spain.

${ }^{\dagger}$ E-mail: leo.gonzalez@upm.es 
The role played by the most commonly used SPH real viscous terms in simulating 2D viscous laminar flows will be discussed. Three viscous terms: Takeda et al. [6], Morris et al. [7] and Monaghan-Cleary-Gingold's $[8,9]$ representing important moments in the history of applications of SPH to viscous flows have being selected. They will be analysed in terms of their conservation properties, and their performance with canonical flows of which the viscosity related phenomena are well understood will be discussed. The dependence of the accuracy of the approximation on the exactness of the derivatives estimation is unquestionable [10-13]. The history of SPH viscosity runs parallel to the approximation of the second-order derivatives that appear in the dissipative terms of the Navier-Stokes equations.

The different case studies were selected for their representativeness of viscosity phenomena with analytical solution and no boundary conditions significantly influencing the flow. Basa $e t$ al. [14] carried out a study focused on the robustness of the SPH formulations for viscous flows using a series of test cases for which no-slip boundary conditions are of paramount importance. Our efforts have being concentrated in the study of the diffusive properties of the fluid itself, which we consider is a crucial problem that should be studied first.

In addition to well-known test cases such as, constant and rigid rotation velocity fields and vortex spin-down, two different simulations of viscous vortex flow dynamics have been selected as the most innovative and difficult of our case studies. From the different vortex models available in fluid mechanics, the Lamb-Oseen viscous vortex model has been selected for these two case studies.

In the first case study, an isolated fixed vortex is diffused in time. The behaviour of an isolated vortex presents a challenge for the SPH viscous terms and its understanding is a necessary step in the study of more complex vortex flows.

In the second case study two co-rotating vortices are transported with diffusion. A flow that is of engineering importance particularly in the modelling of wing tip vortices in Aeronautics. The presence of a second co-rotating vortex complicates greatly the flow of the preceding case. The velocity field of each vortex induces a rotating motion in the other one. The vortices spiral around each other and merge. The combination of the motion with the diffusion process produces an interesting benchmark for the SPH method.

Parallel to the SPH simulations, a finite-element FEM simulation has been performed [15] in both cases and it has been used as a validation result. The computations have been carried out changing the resolution and the domain size with SPH performing very much like the FEM and analytical solutions. In all the case studies the corresponding $2 \mathrm{D}$ flows have been simulated using the three viscosity models and the issues of convergence, stability and accuracy have been addressed.

\section{NAVIER-STOKES CONTINUOUS FORMULATION}

As mentioned in the introduction, our goal is to simulate Newtonian and incompressible viscous flow in laminar regime. These flows are well described in the continuous formulation by the Navier-Stokes equations

$$
\begin{gathered}
\nabla \cdot \mathbf{v}=0 \\
\rho \frac{\mathrm{d} \mathbf{v}}{\mathrm{d} t}=-\nabla P+\mu \nabla^{2} \mathbf{v}
\end{gathered}
$$

in which $P$ is the pressure, $\rho$ the density, $\mathbf{v}$ the velocity and $t$ stands for time.

Equation (1) and the pressure term in Equation (2) play a combined role. The pressure acts as a Lagrange multiplier that produces a zero divergence velocity field. If the incompressibility condition is imposed, either a penalty formulation or a pressure Poisson equation must be solved to calculate the pressure values consequently increasing the computational cost substantially. In the WCSPH context this hypothesis is relaxed by assuming a weakly compressible fluid with a 
large sound speed, where Equation (1) is replaced by

$$
\frac{\mathrm{d} \rho}{\mathrm{d} t}=-\rho \nabla \cdot \mathbf{v}
$$

and a stiff equation of state $P=P(\rho)$ is added to the system.

Finally, assuming the Lagrangian description of the fluid, the fluid particles move according to the kinematic law

$$
\frac{\mathrm{d} \mathbf{r}}{\mathrm{d} t}=\mathbf{v}
$$

where $\mathbf{r}$ is the position vector.

\section{NAVIER-STOKES DISCRETE FORMULATION}

The complete SPH formulation [16] considered will be the following:

$$
\begin{gathered}
\frac{\mathrm{d} \rho_{a}}{\mathrm{~d} t}=\sum_{b \in \mathcal{N}_{a}} m_{b} \mathbf{v}_{a b} \nabla_{a} W_{a b} \\
\frac{\mathrm{d} \mathbf{v}_{a}}{\mathrm{~d} t}=-\sum_{b \in \mathscr{N}_{a}} m_{b}\left(\frac{P_{a}}{\rho_{a}^{2}}+\frac{P_{b}}{\rho_{b}^{2}}\right) \nabla_{a} W_{a b}+\mathbf{\Pi}_{a} \\
\frac{\mathrm{d} \mathbf{r}_{a}}{\mathrm{~d} t}=\mathbf{v}_{a} \\
P=\frac{\rho_{0} c_{s}^{2}}{\gamma}\left(\left(\frac{\rho}{\rho_{0}}\right)^{\gamma}-1\right)
\end{gathered}
$$

in which $m$ is the mass, $\mathbf{r}$ is the position vector, and the subscripts $a$ or $b$ refers to the particle that carries over the considered property.

The notation $\mathbf{v}_{a b}$ means $\mathbf{v}_{a b}=\mathbf{v}_{a}-\mathbf{v}_{b}, \nabla_{a} W_{a b}$ is the gradient of the $b$-centred kernel with respect to the coordinates of particle $a$

$$
\boldsymbol{\Pi}_{a}=-\sum_{b \in \mathscr{N}_{a}} m_{b} \boldsymbol{\Pi}_{a b} \nabla_{a} W_{a b}
$$

is the viscous term at particle $a$, see Equation (8.2) [16], $\mathscr{N}_{a}$ is the index set of particle $a$ neighbours, regarding the kernel support, $\rho_{0}$ the reference density, $c_{s}$ is the numerical sound speed and $\gamma=7$.

The kernel will be a normalized Gaussian kernel, see [17], with a support of $3 h$ and $h=1.33 \mathrm{~d} x$ where $h$ is the smoothing length and $d x$ is the typical initial separation among particles

$$
W\left(r_{a b}, h\right)= \begin{cases}\frac{\mathrm{e}^{-\frac{r_{a b}^{2}}{h^{2}}}-\mathrm{e}^{-9}}{\pi h^{2}\left(1-10 \mathrm{e}^{-9}\right)} & \text { when } r_{a b} \leqslant 3 h \\ 0 & \text { otherwise }\end{cases}
$$

where $\mathbf{r}_{a b}=\mathbf{r}_{a}-\mathbf{r}_{b}$ and $r_{a b}=\left\|\mathbf{r}_{a b}\right\|$ is the Euclidean distance between the two particles.

The integration in time has been performed using a Leap-frog second-order scheme [18]. The selection of the time step has been based on the viscous diffusion, convective, acceleration and sound waves propagation terms [18]. The CFL factor used was $1 / 8$ using $h$ as a reference length. Depending on the case a special initialization or stabilization technique has been used.

Within SPH techniques, WCSPH is the usual way of modelling incompressible free surface flows $[19,20]$. It is easy to programme because the pressure is obtained from a separate equation of state (8) that is chosen so that the speed of sound is large enough to keep the relative density 
fluctuations small [16]. As discussed by Lee et al. [20], a truly incompressible approach gives more accurate values of the pressure than WCSPH. However, when dealing with highly distorted flows the need for an explicit definition of a boundary at the free surface is a major drawback.

\section{VISCOSITY MODELS}

In our analysis we have selected three different SPH implementations of the real viscous terms in the Navier-Stokes linear momentum equation that represent three different important stages in the simulation of SPH physical viscosities.

\subsection{Monaghan-Cleary-Gingold's}

The standard viscous term of Monaghan and Cleary (MCGVT) is based on the linear part of the artificial viscosity that Gingold and Monaghan presented in [21] as the first implementation of shear viscosity for incompressible flows to model real viscosity in shock wave applications

$$
\mathbf{\Pi}_{a}=-\alpha h \sum_{b} \frac{m_{b}}{\bar{\rho}_{a b}} c_{s} \frac{\mathbf{v}_{a b} \cdot \mathbf{r}_{a b}}{r_{a b}^{2}+\xi^{2}} \nabla_{a} W_{a b}
$$

where $\bar{\rho}_{a b}$ is the average density of particles $a$ and $b$, and $\xi$ is a small parameter included to prevent singularities that will not be used in this paper since with a good time-stepping the particles should not get too close.

By converting the summation into integrals the SPH momentum equation was turned back to the continuum. The resulting equation was then compared term by term with the original Navier-Stokes linear momentum equation to give an effective kinematic viscosity for the SPH simulation

$$
v=\frac{\alpha h c}{8}
$$

The final version of MCGVT is then

$$
\mathbf{\Pi}_{a}^{\mathrm{MCGVT}}=-\frac{8 \mu}{\rho_{a}} \sum_{b} \frac{m_{b}}{\rho_{b}} \frac{\mathbf{v}_{a b} \cdot \mathbf{r}_{a b}}{r_{a b}^{2}} \nabla_{a} W_{a b}
$$

The MCGVT conserves linear and angular momenta, it vanishes for rigid rotations and is Galilean invariant [9]. It has been frequently used [2, 14, 22, 23] due to its straightforward implementation from the aforementioned artificial viscosity. Though its consistency for modelling the viscous term of incompressible Newtonian flows was demonstrated by $\mathrm{Hu}$ et al. [23], the radial nature of the MCGVT viscous forces can be seen as a shortcoming when modelling shear forces.

For the Gaussian kernel (9), MCGVT is

$$
\boldsymbol{\Pi}_{a}^{\mathrm{MCGVT}}=-\frac{8 \mu}{\rho_{a}} \sum_{b} \frac{m_{b}}{\rho_{b}} \frac{\mathbf{v}_{a b} \cdot \mathbf{r}_{a b}}{r_{a b}^{2}} \frac{2}{h^{2}} W\left(r_{a b}\right) \mathbf{r}_{a b}
$$

An important step in the evolution of real viscous terms was the Monaghan and Cleary application to heat conduction [8] of the integral approximation of the second order derivative of a magnitude [24], where they produced innovative expressions for the Laplacian which were later extended by Español and Revenga $[9,25]$. Cleary presented in [26] a new form of the viscous diffusion term adapting the estimation of the thermal conduction term in the heat equation

$$
\left(\frac{1}{\rho} \nabla \cdot(\mu \nabla \mathbf{v})\right)_{a}=\sum_{b} \frac{m_{b}}{\rho_{a} \rho_{b}}\left(\mu_{a}+\mu_{b}\right) \mathbf{v}_{a b} \frac{\mathbf{r}_{a b} \cdot \nabla_{a} W_{a b}}{r_{a b}^{2}}
$$

by changing some of its elements to ensure the continuity of the stress tensor across material interfaces and the conservation of the angular momentum. 
The final expression of (CVT) is

$$
\boldsymbol{\Pi}_{a}=-\sum_{b} \xi \frac{m_{b}}{\rho_{a} \rho_{b}} \frac{4 \mu_{a} \mu_{b}}{\mu_{a}+\mu_{b}} \frac{\mathbf{v}_{a b} \cdot \mathbf{r}_{a b}}{r_{a b}^{2}} \nabla_{a} W_{a b}
$$

He introduced a parameter $\xi$ that he estimated numerically, by choosing the value of the parameter that best fits a systematic Couette flows tests as $\xi=4.9633$, instead of $\xi=4$ of the earlier Monaghan's implementation.

When the viscosity is constant $\left(\mu_{a}=\mu_{b}\right)$ the viscous term expression (15) is

$$
\boldsymbol{\Pi}_{a}=-\sum_{b} \xi \frac{2 \mu m_{b}}{\rho_{a} \rho_{b}} \frac{\mathbf{v}_{a b} \cdot \mathbf{r}_{a b}}{r_{a b}^{2}} \nabla_{a} W_{a b}
$$

Using the 2D approximation of second-order derivatives of Español and Revenga [25] we obtain again $\xi=4$ in the case of incompressible flows, consequently CVT coincides with MCGVT (13) in this case.

\subsection{Morris et al.}

Morris et al. [7] made a straightforward use of (14) to estimate the shear viscosity term in the linear momentum Navier-Stokes equation for incompressible flow

$$
\mathbf{\Pi}_{a}^{\mathrm{MVT}}=\sum_{b} \frac{m_{b}}{\rho_{a} \rho_{b}}\left(\mu_{a}+\mu_{b}\right) \mathbf{v}_{a b} \frac{\mathbf{r}_{a b} \cdot \nabla_{a} W_{a b}}{r_{a b}^{2}}
$$

It conserves the linear momentum while the angular momentum is not conserved, a desirable feature of relative importance in low Reynolds number flows and in many industrial fluid problems [9].

When the viscosity is constant and for the Gaussian kernel (9), (MVT) takes the form

$$
\mathbf{\Pi}_{a}^{\mathrm{MVT}}=-\sum_{b} \frac{4 \mu m_{b}}{\rho_{a} \rho_{b}} \frac{W_{a b}}{h^{2}} \mathbf{v}_{a b}
$$

\subsection{Takeda et al.}

When discussing the SPH form of the heat equation Monaghan and Cleary discarded the direct use of second-order derivatives of the smoothing function because it was too sensitive to particle disorder and its application presented some relevant contradictions. Takeda et al. [6] considered the linear momentum equation relative to a compressible Stokesian fluid

$$
\frac{\mathrm{d} \mathbf{v}}{\mathrm{d} t}=-\frac{1}{\rho} \nabla p+\frac{\mu}{\rho}\left(\nabla^{2} \mathbf{v}+\frac{1}{3} \nabla(\nabla \cdot \mathbf{v})\right)
$$

and found their SPH viscous term (TVT) by differentiating the SPH interpolant twice, followed by a subsequent process of anti-symmetrization to recover the conservation of the linear momentum.

Assuming that the kernel is a function of $r_{a b}$, we get the viscous term

$$
\begin{aligned}
\mathbf{\Pi}_{a}^{\mathrm{TVT}}= & \frac{-\mu}{\rho_{a}} \sum_{b} \frac{m_{b}}{\rho_{b}}\left\{\left(r_{a b} \frac{\partial}{\partial r_{a b}}\left(\frac{1}{r_{a b}} \frac{\partial W_{a b}}{\partial r_{a b}}\right)+\frac{2}{r_{a b}} \frac{\partial W_{a b}}{\partial r_{a b}}\right) \mathbf{v}_{a b}+\frac{1}{3}\left[\left(\frac{1}{r_{a b}} \frac{\partial W_{a b}}{\partial r_{a b}}\right) \mathbf{v}_{a b}\right.\right. \\
& \left.\left.+\frac{\partial}{\partial r_{a b}}\left(\frac{1}{r_{a b}} \frac{\partial W_{a b}}{\partial r_{a b}}\right) \frac{\mathbf{r}_{a b} \cdot \mathbf{v}_{a b}}{r_{a b}} \mathbf{r}_{a b}\right]\right\}
\end{aligned}
$$

where each term of the sum has been split into one term linked to the shear viscosity, the one parallel to $\mathbf{v}_{a b}$ that explicitly reproduces in the discrete formulation the role played by the Laplacian in the continuum, and a second one representing the compressible viscosity.

The final expression of TVT for the renormalized Gaussian kernel (9), is

$$
\mathbf{\Pi}_{a}^{\mathrm{TVT}}=\frac{-\mu}{\rho_{a}} \sum_{b} \frac{m_{b}}{\rho_{b}}\left[\frac{7}{3}\left(-\frac{2 W_{a b}}{h^{2}}\right) \mathbf{v}_{a b}+\left(\frac{\mathbf{v}_{a b} \cdot \mathbf{r}_{a b}}{3} \mathbf{r}_{a b}+\mathbf{v}_{a b} r_{a b}^{2}\right) \frac{4 W_{a b}}{h^{4}}\right]
$$


The algebraic structure of TVT makes the conservation of the angular momentum impossible; however, their results are very accurate and although it is not often used it remains an important contribution in the evolution of the physical viscous terms.

\section{TESTS}

\subsection{General}

In order to assess the performance of the three viscous models under study, a series of test cases with increasing difficulties in their physics have been designed. They correspond to some canonical flows in Fluid Dynamics as well as representative flows in Engineering that will help to show the advantages and limitations of the considered viscous models. They are the following ones:

(1) Constant and rigid rotation velocity fields. The viscous efforts associated with these fields must vanish.

(2) Vortex spin-down. A case that was studied by Monaghan [9] using the MCGVT. The study will be extended here to the (MVT) and the (TVT).

(3) Isolated Lamb-Oseen vortex evolution. A flow of engineering importance particularly in the modelling of wing tip vortices in Aeronautics. The behaviour of an isolated vortex presents a challenge for the SPH viscous terms and its understanding is a necessary step in the study of more complex vortex flows. In this case, the accuracy of the differential operators will be directly studied for the initial conditions of the flow. The results of the SPH numerical simulation will be compared with the exact solution and with a FEM numerical solution that has been obtained for this purpose.

(4) Co-rotating vortex pair evolution. The presence of a second co-rotating vortex complicates greatly the flow of the preceding case. The velocity field of each vortex induces a rotating motion in the other one. The vortices spiral around each other and merge. This motion is also combined with the typical diffusion process producing a interesting benchmark for the SPH method. The results of the SPH numerical simulation will be compared with an FEM numerical solution that has been developed for this case.

\subsection{Constant velocity}

The continuous differential operators that model the viscous forces are identically null for a constant velocity field. The discretized versions of those operators do not in general respect this principle (Galilean invariance) when a large constant velocity field is part of the flow, see for instance [27]. It is therefore relevant to discuss how the three viscous terms here considered behave under these conditions. The discussion is trivial for these particular formulations since all of them depend explicitly on $\mathbf{v}_{a b}$, and consequently the viscous efforts associated with the constant velocity field vanish.

\subsection{Rigid rotation}

5.3.1. Setup. The behaviour of the three considered viscosity models will be tested here with a $2 \mathrm{D}$ velocity field defined by a rigid rotation. This velocity field can be expressed as $\mathbf{v}(\mathbf{r})=\boldsymbol{\Omega} \times \mathbf{r} \rightarrow$ $\mathbf{v}(x, y)=\Omega \mathbf{k} \times(x \mathbf{i}+y \mathbf{j})$. Considering for simplicity $\Omega=1$, we get $\mathbf{v}(x, y)=u \mathbf{i}+v \mathbf{j}$ with $u=-y$ and $v=x$.

As it is well known, a rigid rotation velocity field is a particular solution of the incompressible Navier-Stokes equations when the fluid moves inside a rotating cylinder with constant angular velocity $\Omega$, see [28]. Assuming that the rigid rotation $\mathbf{v}$ is the initial velocity field of a flow, we will study whether the WCSPH formulation is able to accept a rigid rotation velocity field as a particular solution or not. We would also like to examine if the rigid rotation is a stationary solution of our WCSPH formulation or if it decays/blows up as time evolves.

In this study each one of the previously described viscosity model will be considered. The equation of state that will be used is (8). This equation of state has the advantage of removing 
spurious boundary effects in free surface flows [7], but it is also responsible for creating numerical instabilities in regions of sustained low pressure. Another important parameter in the problem is the initial density of the fluid $\rho_{0}=\rho_{\text {ref }}+\Delta \rho$. For the present calculation $\rho_{\text {ref }}=1$ and the sound speed has been taken as 10 , which is 10 times the maximum velocity of the rigid rotation. Depending on whether $\Delta \rho$ is zero or not, a tensile instability problem could appear in the fluid turning the problem into an unstable problem, see [29].

In order to analyze this case, three tests with different equations of state and initial densities will be conducted.

- Equation of state (8), $\triangle \rho=0.02$, and initial density $\rho_{0}=1.02$.

- Equation of state (8), $\triangle \rho=0.0$, and initial density $\rho_{0}=1.0$.

- Initial density $\rho_{0}=\rho_{\text {ref }}$, but the Equation (8) has been substituted by:

$$
P=c_{s}^{2} \rho
$$

As a measure of the error between the WCSPH simulation and the analytical solution of the incompressible Navier-Stokes equations $\mathbf{v}=\left(v_{r}, v_{\theta}\right)=(0, \Omega r)$, where $v_{r}$ and $v_{\theta}$ are the radial and tangential velocity components, the velocity relative error RE

$$
\mathrm{RE}=\frac{\sqrt{v_{r}^{2}+\left(v_{\theta}-\Omega r\right)^{2}}}{\Omega r}
$$

will be used.

In the initial configuration, a number of particles of equal mass were placed on concentric rings in the circular domain, starting with one particle at the centre. These rings were equally spaced with spacing $\triangle p=R / n r$, where $R$ is the radius of the domain and $n r$ represents the number of rings (the ring density). For the present case $R=1$. The particles were equally spaced around the rings and the number of particles placed at the ring of radius $r$ was $n p(r)=\operatorname{nint}(2 \pi r / \Delta p)$, where 'nint' means the nearest integer number, see Figure 1. Starting with a minimum of $n r=22$, an increasing number of rings were used in the simulations to test the convergence properties.

In these simulations, the last three rows of boundary fluid particles are placed outside the domain and form the rotating boundary. Two different cases were considered. In the first case the boundary particles have prescribed trajectories, while in the second case they are fixed. In both cases these particles have prescribed tangential velocity $\Omega r$. In all the case studies, the density and pressure are kept constant at the boundary particles.

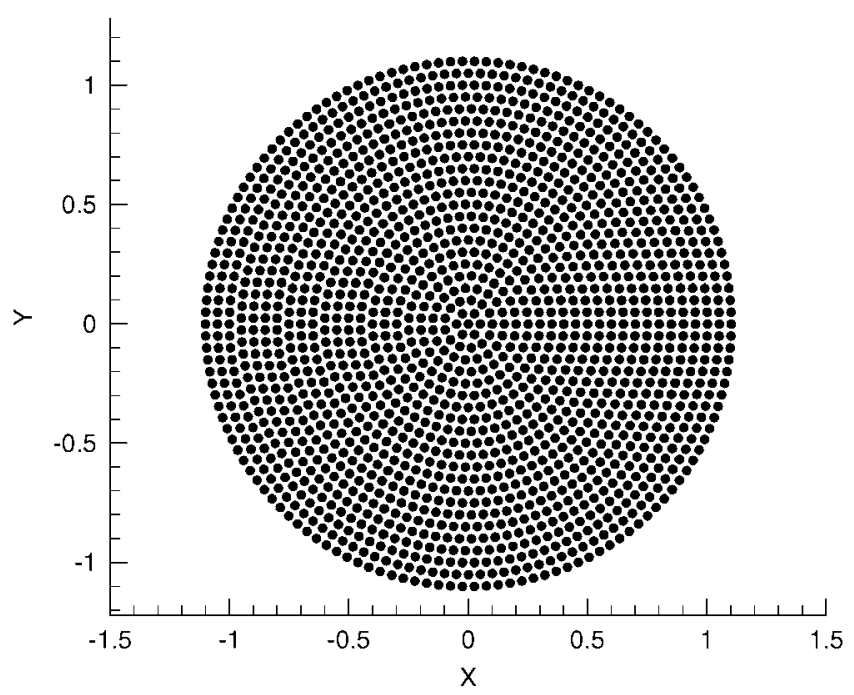

Figure 1. Initial setup of the particles for the rigid rotation test when $n r=22$. 
Table I. Modulus of the non-dimensional viscous force calculated in the initial state by the MVT and TVT viscous models for the rigid rotation velocity field $(u, v)=(-y, x) . h / d x=1.33$.

\begin{tabular}{lcc}
\hline$n r$ & $\Pi_{a \mathrm{MVT}} \rho_{a} h / 4 \Omega \mu$ & $\Pi_{a}$ TVT $\rho_{a} h / \Omega \mu$ \\
\hline 10 & 0.36595911223729 & 0.67122432226925 \\
20 & 0.00000355946777 & 0.00005032325801 \\
30 & 0.00000000000338 & 0.00000000009584 \\
\hline
\end{tabular}

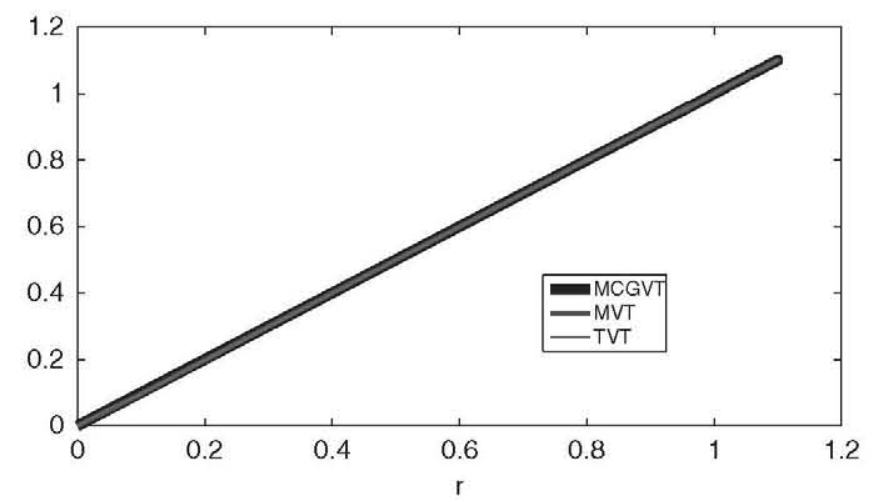

Figure 2. $V_{\theta}$ versus $r$ in the rigid rotation test for the three viscosity models at time $t=4 \pi$ ( 2 complete laps) when the boundary fluid particles have fixed positions. Equation of state (8), $n r=22$ rings and initial density $\rho_{0}=1.02$.

The results do not show important differences between these two cases. Consequently for the sake of clarity, only those corresponding to the fixed boundary fluid particles have been included in the following section.

5.3.2. Results. In the MCGVT (13), the dot product $\mathbf{v}_{a b} \cdot \mathbf{r}_{a b}$, is in this case the mixed product $\left(\boldsymbol{\Omega} \times \mathbf{r}_{a b}\right) \cdot \mathbf{r}_{a b}$ that always vanishes.

In Morris' viscosity model we evaluate in the initial state the non-dimensional form $\Pi_{a}^{\mathrm{MVT}} \rho_{a} h / 4 \Omega \mu$. The results, that are presented in the first column of Table I, show that with a linear velocity field, the MVT calculates the viscous term accurately.

Finally, Takeda's viscosity model TVT (21) is for the rigid rotation velocity field

$$
\boldsymbol{\Pi}_{a}^{\mathrm{TVT}}=\frac{-\mu}{\rho_{a}} \sum_{b} \frac{m_{b}}{\rho_{b}}\left(\frac{7}{3}+2 r_{a b}^{2}\right) \frac{2 W_{a b}}{h^{2}} \mathbf{v}_{a b}
$$

The values in the initial state of the non-dimensional form $\mathbf{I}_{a}^{\mathrm{TVT}} \rho_{a} h / \Omega \mu$ are presented in the second column of Table I. As in Morris' model, Takeda's model reproduces the viscous dissipation accurately.

Figure 2 shows clearly that the WCSPH formulation of the three considered viscous models, using the equation of state (8) and $\Delta \rho=0.02$, reproduces correctly a rigid rotation velocity field when the boundary fluid particles are fixed. The presence of clumping for high values of the radius is due to the centrifugal force caused by the rotation. When the particles start rotating, a centrifugal force pushed them towards the boundary until it is balanced by a reacting force. The result of this process is that most of the fluid particles suffer an increasing radial displacement producing a slight clumping near the boundary. As a consequence, a radial pressure gradient is created which finally equilibrates the system. The increment of the number of rings does not change the result significantly, showing that the WCSPH formulation converges to the exact solution when the total number of particles is increased. 
Table II. Maximum relative error $\operatorname{Max} R E$ and the corresponding radius $r(\operatorname{Max} R E$ ) where the maximum relative error is located in the rigid rotation test. Different viscosity models are evaluated at time $t=4 \pi$ for different combinations of boundary conditions ( $\mathrm{fb}$ : fixed boundary, mpb: moving prescribed boundary) and resolutions. The equation of state (8) and $\triangle \rho=0.02$ are used.

\begin{tabular}{lcccc}
\hline Viscous model & Max RE & $r$ (Max RE $)$ & $n r$ & Boundary condition \\
\hline MCGVT & $3.28 \mathrm{e}-3$ & 0.9411 & 22 & $\mathrm{fb}$ \\
MCGVT & $3.28 \mathrm{e}-3$ & 0.9411 & 22 & $\mathrm{mpb}$ \\
MCGVT & $5.96 \mathrm{e}-3$ & 0.0234 & 44 & $\mathrm{fb}$ \\
MCGVT & $5.96 \mathrm{e}-3$ & 0.0234 & 44 & $\mathrm{mpb}$ \\
MCGVT & $3.01 \mathrm{e}-3$ & 0.0358 & 88 & $\mathrm{fb}$ \\
MVT & 0.0570 & 0.0508 & 22 & $\mathrm{fb}$ \\
MVT & 0.0565 & 0.0507 & 22 & $\mathrm{mpb}$ \\
MVT & 0.0587 & 0.0229 & 44 & $\mathrm{mpb}$ \\
MVT & 0.0579 & 0.0231 & 44 & $\mathrm{fb}$ \\
MVT & 0.0553 & 0.0107 & 88 & $\mathrm{mpb}$ \\
TVT & 0.0543 & 0.0985 & 22 & $\mathrm{fb}$ \\
TVT & 0.0531 & 0.0996 & 22 & $\mathrm{mpb}$ \\
TVT & 0.0511 & 0.0470 & 44 & $\mathrm{fb}$ \\
TVT & 0.0486 & 0.0467 & 44 & $\mathrm{mpb}$ \\
TVT & 0.0564 & 0.0225 & 88 & $\mathrm{fb}$ \\
\hline
\end{tabular}

Table III. Maximum relative error Max RE and the corresponding radius $r$ (Max RE) where the maximum relative error is located in the rigid rotation test. Different viscosity models are evaluated at time $t=4 \pi$ for different combinations of boundary conditions (fb: fixed boundary, mpb: moving prescribed boundary) and resolutions. The equation of state (8) and $\triangle \rho=0.0$ are used.

\begin{tabular}{lcccc}
\hline Viscous model & Max RE4 & $r$ (Max RE) & $n r$ & Boundary condition \\
\hline MCGVT & 1.0257 & 0.0131 & 22 & $\mathrm{fb}$ \\
MCGVT & 1.0257 & 0.0131 & 22 & $\mathrm{mpb}$ \\
MCGVT & 0.0503 & 0.0946 & 44 & $\mathrm{fb}$ \\
MCGVT & 0.0503 & 0.0946 & 44 & $\mathrm{mpb}$ \\
MCGVT & $6.14 \mathrm{e}-3$ & 0.0011 & 88 & $\mathrm{fb}$ \\
MVT & 0.7122 & 0.0701 & 22 & $\mathrm{fb}$ \\
MVT & 0.7373 & 0.0687 & 22 & $\mathrm{mpb}$ \\
MVT & 0.9916 & 0.0541 & 44 & $\mathrm{mpb}$ \\
MVT & 0.9313 & 0.0647 & 44 & $\mathrm{fb}$ \\
MVT & 0.0594 & 0.0104 & 88 & $\mathrm{fb}$ \\
TVT & 9.5981 & 0.0451 & 22 & $\mathrm{fb}$ \\
TVT & 6.5239 & 0.0643 & 22 & $\mathrm{mpb}$ \\
TVT & 27.664 & 0.0157 & 44 & $\mathrm{fb}$ \\
TVT & 15.817 & 0.0120 & 44 & $\mathrm{mpb}$ \\
TVT & 0.1951 & 0.0011 & 88 & $\mathrm{fb}$ \\
\hline
\end{tabular}

In Table II the maximum of the velocity relative error RE at time $t=4 \pi$ ( 2 complete laps) for the three viscosity models is compared when the equation of state (8) is used and $\triangle \rho=0.02$. In all cases, the error is less than $6 \%$ and even smaller for the MCGVT viscous model.

The same maximum of the relative error $R E$ at time $t=4 \pi$ for the three viscosity models and equation of state (8) but with a different initial density increment $\triangle \rho=0$ is compared in Table III. These results show that for certain resolutions an instability appears in the fluid producing a large error in the velocity calculation. This error comes from the fact that the initial pressure is zero when $\triangle \rho=0$, and this low pressure creates certain instability in the fluid making the central rings gathering into couples. The error decreases when the number of rings is increased up to $n r=88$, see Table III.

The tensile instability phenomenon can be removed from this problems using an equation of state different than the one proposed in (8). The change of the equation of state eliminated all the 
Table IV. Maximum relative error versus $r$ in the rigid rotation test for the different viscosity models at time $t=4 \pi$. The equation of state (22) and $\rho_{0}=\rho_{\text {ref }}$ are used.

\begin{tabular}{lccc}
\hline Viscous model & Max RE & $r$ (Max RE) & $n r$ \\
\hline MCGVT & 0.5784 & 0.0125 & 22 \\
MCGVT & 0.3097 & 0.0434 & 44 \\
MCGVT & $2.14 \mathrm{e}-3$ & 0.0023 & 88 \\
MVT & 0.9162 & 0.0718 & 22 \\
MVT & 0.3313 & 0.0516 & 44 \\
MVT & 0.0292 & 0.0144 & 88 \\
TVT & 0.9815 & 0.0374 & 22 \\
TVT & 0.6654 & 0.0147 & 44 \\
TVT & 0.0808 & 0.0157 & 88 \\
\hline
\end{tabular}

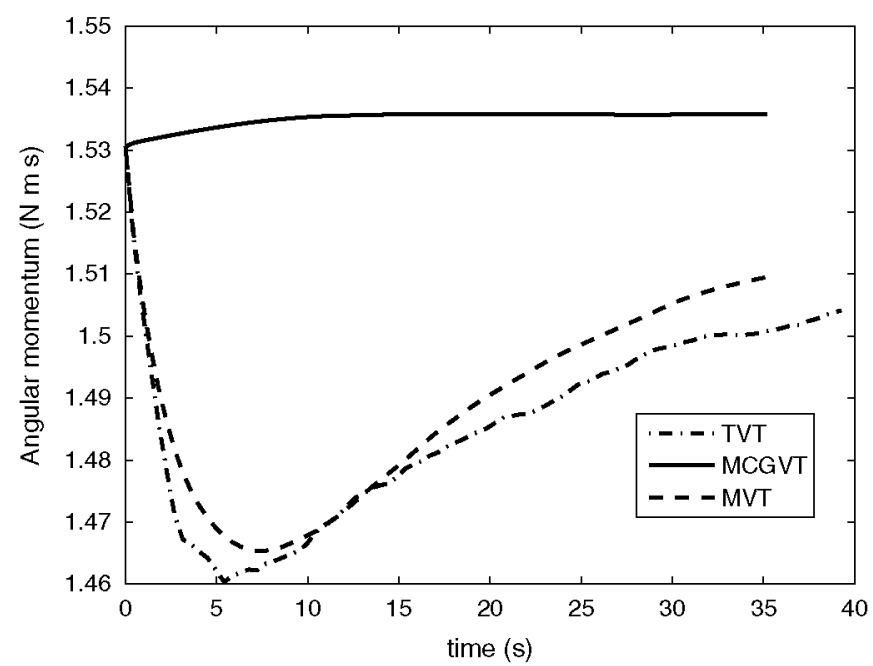

Figure 3. Angular momentum evolution in the rigid rotation test for the three different viscosity models for $n r=44$ rings.

instabilities appearing in the centre of the domain as expected. Repeating computations analogous to the ones shown in Table III, with the new equation of state (22) and $\rho_{0}=\rho_{\text {ref }}$, gave significantly improved results, see Table IV. This result shows the influence of the equation of state used in the problem on the tensile instability, see [7].

In the continuous limit a rotating disc with constant angular velocity $\Omega=1$, unit density $\rho=1$ and radius $R=1$ has a global angular momentum $L=\pi \rho \Omega R^{4} / 2=\pi / 2=1.57$. The convergence of the initial angular momentum to this value when the number of particles is increased has also been examined.

The behaviour of the different viscous terms with respect to the angular momentum conservation property in the absence of viscous interaction between the fluid particles and when the boundary fluid particles and the pressure force is the only reaction coming from the boundary has been investigated. Figure 3 represents the evolutionary curves of the angular momentum of the different viscous terms when $n r=44$. The only one that keeps a constant global angular momentum of the system is the MCGVT. The value of the constant is a good approximation to the analytical value $\pi / 2=1.57$. The other viscous term do not tend to any asymptotic value.

It is interesting to note a slight increment of the angular momentum with time in the MCGVT evolutionary curve due to the compression effect produced by the before mentioned centrifugal force at high radius values that pushes the particles to the outer regions where their larger velocities and radii, increase the angular momentum asymptotically in time. In Figure 4 the sum of the radii 


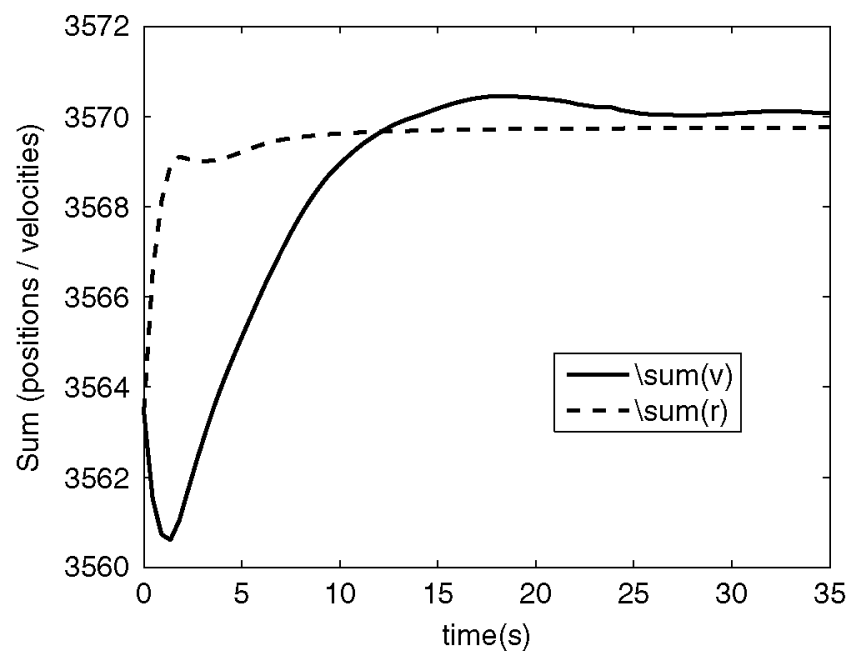

Figure 4. Long-term representation of the total velocity and total radius versus time in the rigid rotation test for the MCGVT viscous model. $n r=44$ rings.

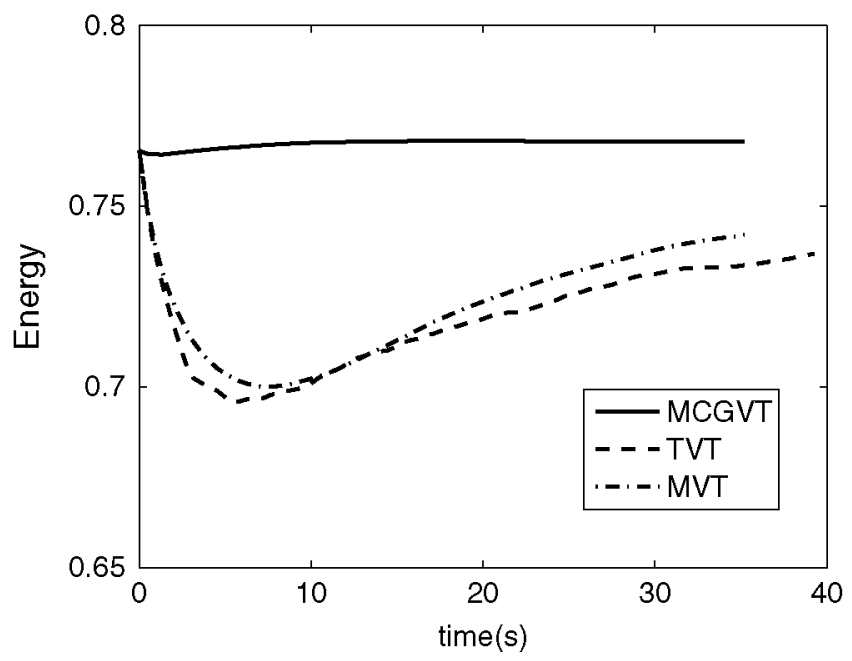

Figure 5. Kinetic energy evolution in the rigid rotation test for the three different viscosity models for 44 rings.

and the sum of the velocities of all particles have been monitored versus time. A similar asymptotic behaviour can be observed in both magnitudes that as a result produce the angular momentum convergence.

Similarly, the time evolution of the kinetic energy has also been studied. The corresponding evolutionary curves for $n r=44$ are represented in Figure 5. Once again the MCGVT is the only viscous term capable of conserving the kinetic energy. The asymptotic constant value of the kinetic energy is in agreement with the analytical value $K=\pi \rho \Omega^{2} R^{4} / 4=\pi / 4=0.78$. The slight increment in the time-energy curve can be justified by the presence of the centrifugal force.

\subsection{Vortex spin-down}

5.4.1. Setup. In response to a work of Imaeda and Inutsuka [30], Monaghan published in 2005 an extensive paper on shear flows [9]. Imaeda and Inutsuka had questioned the ability of SPH to model certain shear flows due to some problems in accurately computing the density field. Monaghan [9] tried to refute their conclusions and proposed a series of test cases. One of them, 

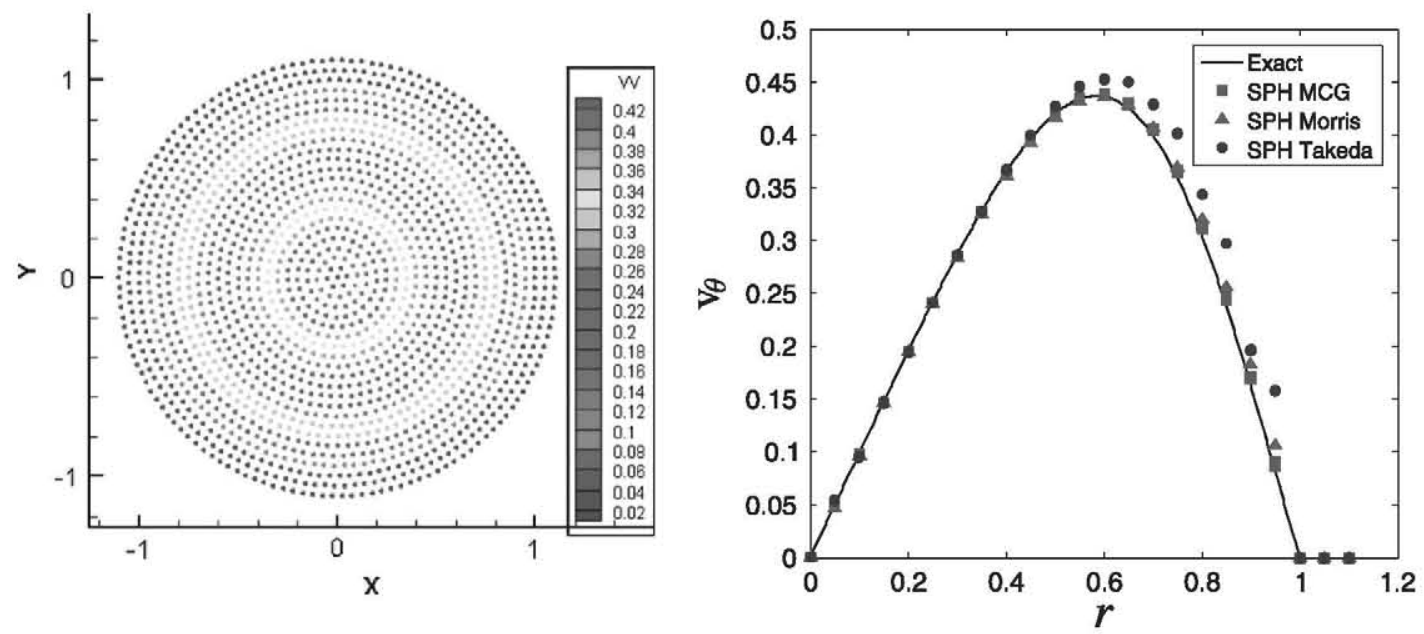

Figure 6. Vortex Spin-down [9], time 1.14s. Velocity field for the MCG term. Radial distribution of the velocity field.

the spin-down of an initially rigidly rotating fluid in a circular 2D container using the MCGVT, is of special interest for our paper where this study will be extended to the MVT and the TVT. In this problem, the time rate of the component of the velocity can be obtained from the formula given by Batchelor [28]. The outmost particle layers are suddenly stopped and the fluid slows its motion due to the shear produced by the outer rings of the region. Structurally, it is like imposing a no-slip boundary condition between the still outmost rings and the bulk of the fluid. These no-slip conditions are not part of our study but they are necessary in this particular case.

It is important to remark that a jump discontinuity in the initial velocity field between the boundary and the moving particles produces a large change in the time rate of density $\mathrm{d} \rho / \mathrm{d} t$ due to its close dependance on the relative velocity between neighbouring particles. These density variations provoke important instabilities in the code, as a result, in order to prevent these artificial density changes the initial velocity field should be continuous.

5.4.2. Results. The agreement of the results with the exact solution is satisfactory for the MCGVT and MVT and not so for the TVT, as can be seen in Figure 6, where the same resolution used by Monaghan [9] $n r=22$, has also been used here.

It is interesting to check to what extent the results in the TVT are affected by the presence of terms coming from the compressible part of the model. To do so, the compressible terms were removed from the TVT equation (21) to get

$$
\mathbf{I}_{a}^{\mathrm{TVT}}=\frac{-\mu}{\rho_{a}} \sum_{b} \frac{m_{b}}{\rho_{b}} \frac{4 W_{a b}}{h^{4}}\left(r_{a b}^{2}-h^{2}\right) \mathbf{v}_{a b}
$$

The convergence properties of this problem fall under the scope of the paper. Consequently, the dependance of Monaghan's result [9] on the number of rings when $h / \mathrm{d} x=1.33$ will be investigated. As Figure 7 shows, when the number of rings is increased, the error of the solution tends to an asymptotic value for all the viscosity models. In this case, there is a significant difference between the three viscous models, while the MTV and the TVT have a decreasing error, the MCGVT has an error that increases with $n r$. Generally speaking, there are two error sources coming from the viscous term calculation, the discretization of the integral formulation via a quadrature formula, and the kernel approximation [13]. The corresponding errors depend basically on both the smoothing length $h$ and the ratio of particle spacing to smoothing length $h / \mathrm{d} x$ and both should tend to zero simultaneously to eliminate the numerical error. The total error can also be influenced by boundary conditions [31] that are not considered by Quinlan et al. [13]. In our calculation $h / \mathrm{d} x$ is kept constant and no-slip boundary conditions are imposed, therefore the increment of the number of 


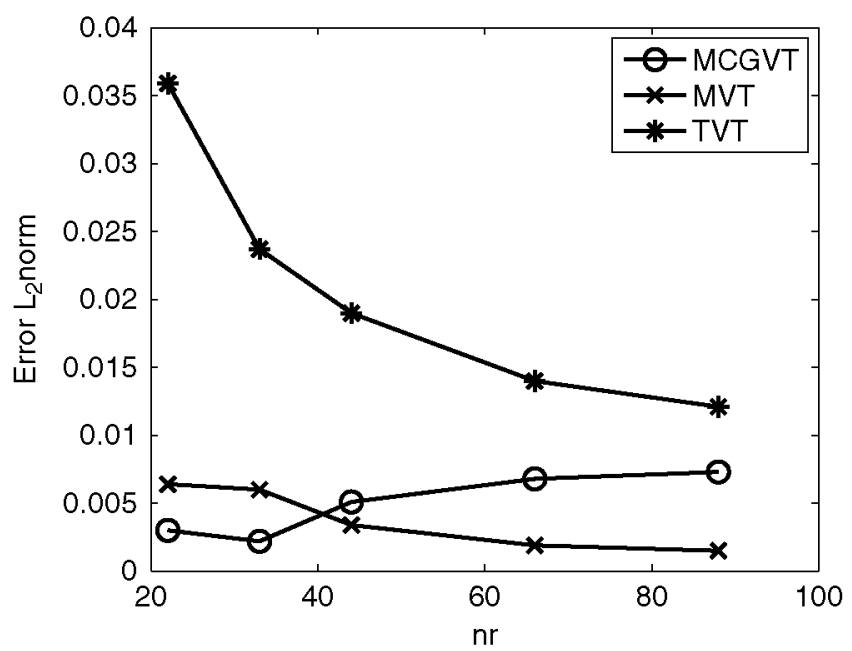

Figure 7 . Convergence study of velocity versus radius at time $1.14 \mathrm{~s}$ for the spin-down vortex with the three viscosity models. $h / d x=1.33$.

particles does not imply numerical convergence. In fact, when the particles are distributed nonuniformly and the boundary conditions play a relevant role on the physics of the problem, the convergence properties change significantly and in some cases, the error can grow when $\mathrm{d} x$ tends to zero or $n r$ increases, see [13].

\subsection{Lamb-Oseen vortex evolution}

In this section the pure diffusion process of an isolated vortex-type flow in an infinite domain will be considered. The initial vorticity of this flow is concentrated in the centre of the domain and decays exponentially towards the boundaries. Therefore, if the boundary conditions are imposed at infinity they will have irrelevant influence on the physics. Among the different available vortex models, the Lamb-Oseen viscous vortex model was selected. Assuming an unidirectional and axisymmetric velocity field $v_{\theta}(r, t)$ and appropriate boundary and initial conditions, see Appendix A for details, the Lamb-Oseen vortex is the simplest vortex solution to the $2 \mathrm{D}$ Navier-Stokes equations. The temporal evolution of an isolated Lamb-Oseen vortex has a time-linearly increasing viscous dependence through the square of the effective vortex core radius $a^{2}$ (maximum velocity radius)

$$
a^{2}(t)=a_{0}^{2}+4 v t
$$

where $a_{0}$ and $v$ denote the initial vortex core radius and the kinematic viscosity, respectively.

These types of vortices are frequently used to model the evolution and stability of aircraft wakes which are composed of an external vortex pair (modelling the wing tip vortices) and an internal vortex pair rotating in the opposite direction (modelling the vortices generated by the fuselage and the horizontal tail) [32].

The centre of the vortex is taken as the origin of the coordinate system with its initial core radius $r_{0}=1$. The initial vorticity field is

$$
\omega_{0}(\mathbf{x}):=2 q \mathrm{e}^{-\left(|\mathbf{x}| / r_{0}\right)^{2}} \quad \text { with } \mathbf{x} \in \mathbb{R}^{2}
$$

where $q$ is the strength of the vortex.

The corresponding velocity field $\left(\nabla \times \mathbf{v}_{\mathbf{L} \mathbf{O}}=\omega_{0}\right)$

$$
\mathbf{v}_{\mathbf{L O}}=(u, v)=q \frac{1.0-\mathrm{e}^{-|\mathbf{x}|^{2}}}{|\mathbf{x}|^{2}}(-y, x)
$$

will be imposed as the initial condition in our code. 
Table V. (Lamb-Oseen vortex evolution) Simulation cases for each viscosity term (for the Lamb-Oseen vortex evolution).

\begin{tabular}{lcclc}
\hline ID & Domain & Diameter & $d x$ & Particles \\
\hline 1 & Circle & 12.4 & 0.2 & 3509 \\
2 & Circle & 12.4 & 0.1 & 13038 \\
3 & Circle & 12.4 & 0.05 & 50209 \\
4 & circle & 24.8 & 0.2 & 13038 \\
5 & Circle & 24.8 & 0.1 & 50209 \\
6 & Circle & 49.6 & 0.2 & 50209 \\
7 & Square & 24.8 & 0.2 & 16641 \\
8 & Square & 24.8 & 0.1 & 64009 \\
9 & Square & 24.8 & 0.05 & 251001 \\
\hline
\end{tabular}

The circulation of the vortex is $\Gamma=2 \pi q$. The Reynolds number $R e$ characterizing the viscous regime will be defined as

$$
R e=\frac{\Gamma}{v}
$$

We take $q=0.5$ and fix $R e=10$, so that the vortex remains stable and the flow is laminar.

In Appendix A an explicit analytical solution for this problem has been found. The exact vorticity time evolution, the maximum velocity and the radius of the location of maximum velocity derived from the analytical solution, will be compared with the SPH numerical solution. This problem has also been solved numerically using a finite-element FEM numerical simulation [15]. The aim of finding a numerical solution using a well-established technique for laminar flows was to compare the SPH simulation not only with an analytical solution but also with another numerical result.

In regard to the SPH computations, circular and square domains have been considered. Since the analytic problem is defined in an unbounded domain with asymptotic boundary conditions (see Appendix A), while the computational domain is finite, a study of convergence of the results with respect to the size of the domain has been performed.

At the boundary of the domain, four rows of fixed fluid particles have been placed. These boundary particles are similar to the ordinary fluid particles with the special feature that they have zero velocity through the whole simulation and consequently they are not displaced at the end of the time step. For each domain size, the resolution has also been changed. The sound speed was taken as 5, which is 10 times the exact maximum velocity of the analytical solution. The results will be presented in terms of the velocity field, comparing the maximum velocity versus time curves for the three terms with the FEM and exact solutions. ${ }^{\ddagger}$

The experiments begin considering a circular (resp. square) domain with a diameter (resp. side length) $D$ equals 12.4 and a particle spacing $\mathrm{d} x$ of 0.2 . The resolution is doubled fixing $\mathrm{d} x=0.1$ and 0.05 . The diameter (resp. side length) of the domain will also double. The relation between the resolution $\mathrm{d} x$, the diameter $D$ and the number of rows $n r$ can be approximated by $\mathrm{d} x=D /(2 n r)$. The simulations run during $10 \mathrm{~s}$ which corresponds to a maximum vorticity of 0.2 (see Appendix A for details) with each one of the three viscosity models discussed in the paper. The matrix of the performed simulations can be seen in Table V. Simulation numbers ( 4 and 5) will be repeated for a square domain ( 7 and 8$)$.

A converged result in terms of the velocity for an increasing particle resolution and for any of the viscosity models has been obtained. See Figure 8 . As in the spin-down test, when the number of rings is increased the error of the solution tends to an asymptotic value for all viscosity models. The lack of good convergence properties for the three viscous models, when $n r$ is increased and $h / \mathrm{d} x$ is kept constant, is due to the non-uniformity of the particle distribution and the lack of

\footnotetext{
${ }^{*}$ In principle it is feasible to obtain the evolution of the vorticity field. However, this would require the differentiation of the velocity field, a process that will induce undesired errors.
} 


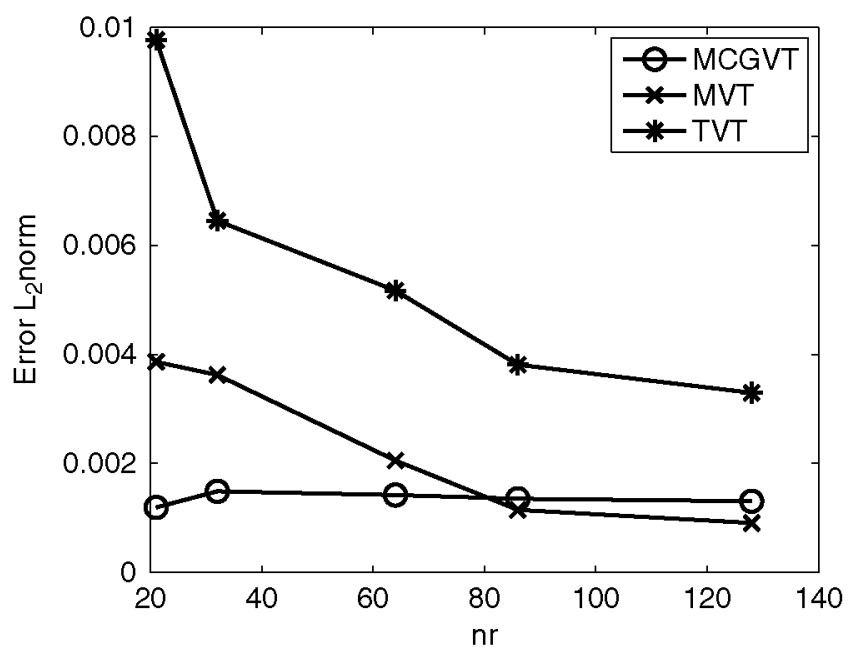

Figure 8. Convergence test of velocity versus radius curve for varying particle resolutions in the Lamb-Oseen vortex evolution at $t=1$ for $D=24.8$.
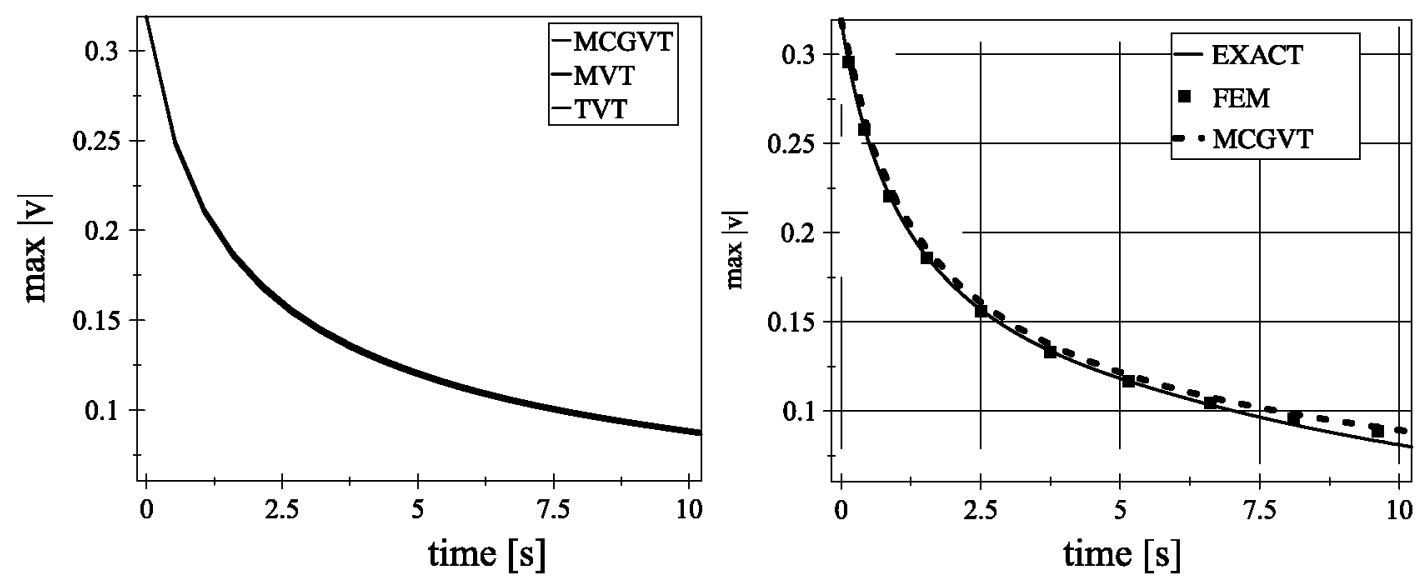

Figure 9. Lamb-Oseen vortex, comparison of the maximum velocity of the viscous models with the corresponding of the exact and FEM solutions. Left: viscous models comparison, right: MCGVT versus FEM and exact solution.

consistency of the SPH formulation, see [13]. Comparing this case with the spin-down case a slight change in the convergence is appreciated as a consequence of the irrelevant role played by the boundary conditions in the evolution of the isolated vortex.

Resolution convergence has been explored running the simulations with $\mathrm{d} x=0.05,0.1,0.2$. The convergence is good for all the viscous terms considering the maximum velocity versus time curves as convergence indicators.

The same indicator was used to assess convergence keeping the resolutions and increasing the domain size. Within the time range chosen for this paper (around 10 time units), the results for the smallest domain size $(D=12.4)$ differ significantly from the results of the other two domain sizes, which fully agree between them. Therefore, the $D=12.4$ domain is not large enough and results regarding the accuracy of the model will be presented for the mid-size domain $D=24.8$.

The results of the comparison of the three viscosity models with the exact and FEM solutions, for the mid-resolution and $D=24.8$, are presented in Figure 9 which shows clearly that the three models give the same results, with correct accuracy.

The results for a square domain show exactly the same trends as before, as is shown in Figures 10 and 11 , where they are compared with the exact and FEM solutions. 


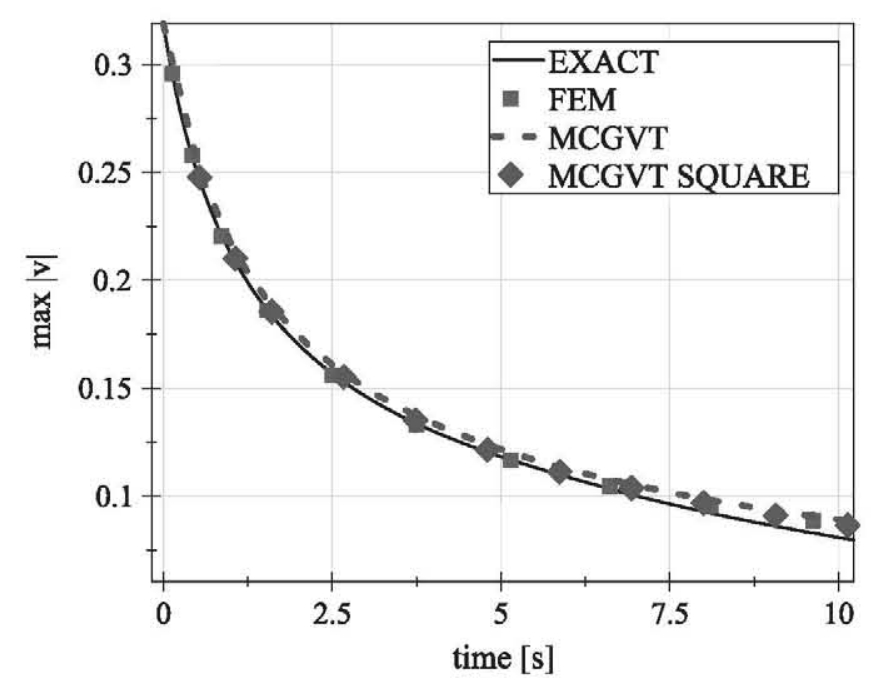

Figure 10. Lamb-Oseen vortex maximum velocity versus time curve for $D=24.8, \mathrm{~d} x=0.1$, square and circular domains.
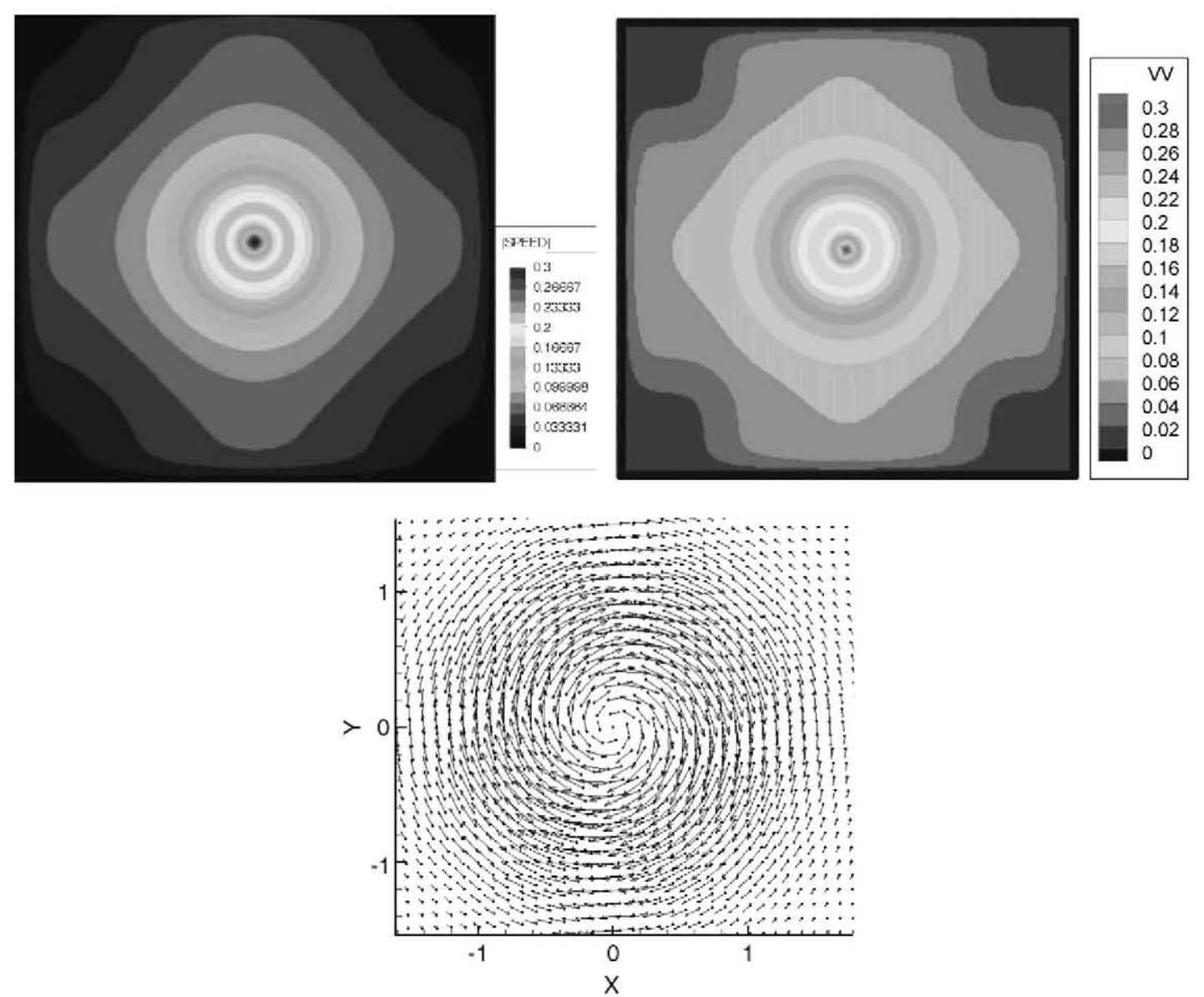

Figure 11. Lamb-Oseen vortex, $R e=10$. Top left: velocity modulus field at $t=1 \mathrm{~s} \mathrm{FEM}$; top right: Idem, MCGVT; bottom: MCGVT, velocity vectors in the vortex core. 

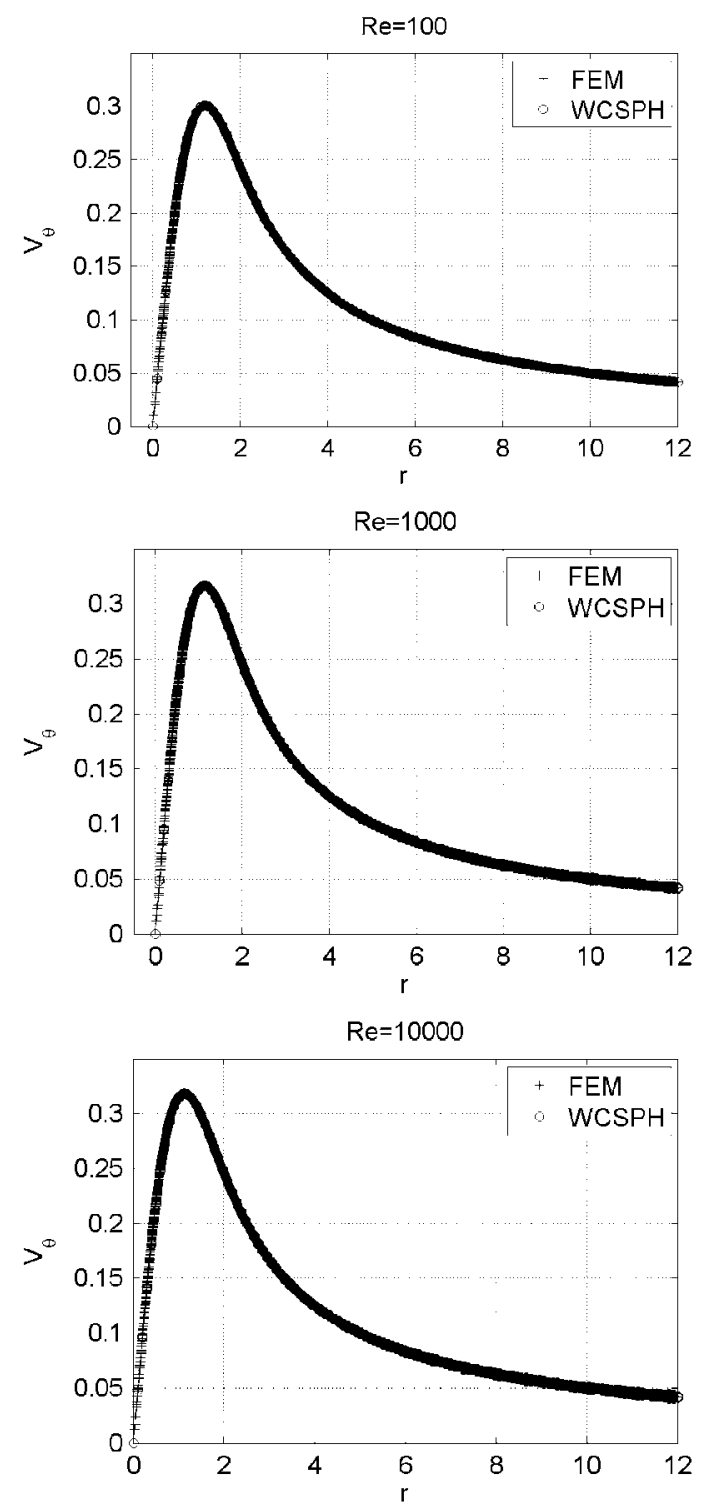

Figure 12. Comparison of the velocity magnitude between the WCSPH(MCGVT) solution and the FEM code for the Lamb-Oseen vortex diffusion at $t=1$ for different Reynolds numbers (Diameter $D=24.8$ ).

Once the validation has been done at low Reynolds numbers $R e=10$, it is convenient to verify if the agreement is valid for increasing Reynolds numbers. The answer is affirmative as indicated by Figure 12 in which similar tests for increasing Reynolds numbers $R e=100,1000,10000$ with the MCGVT model are represented.

\subsection{Co-rotating vortex dipole}

The pairing of same sign vortices (co-rotating vortices) is believed to be the dominant process of $2 \mathrm{D}$ turbulence. The dynamics of co-rotating Lamb-Oseen vortex pair is much more complex than the preceding one, mainly because of the lack of a dominant direction of the velocity field in the domain. In the co-rotating vortex pair, the self-induced rotation of the vortex pair results in a complex velocity field with different directions. 

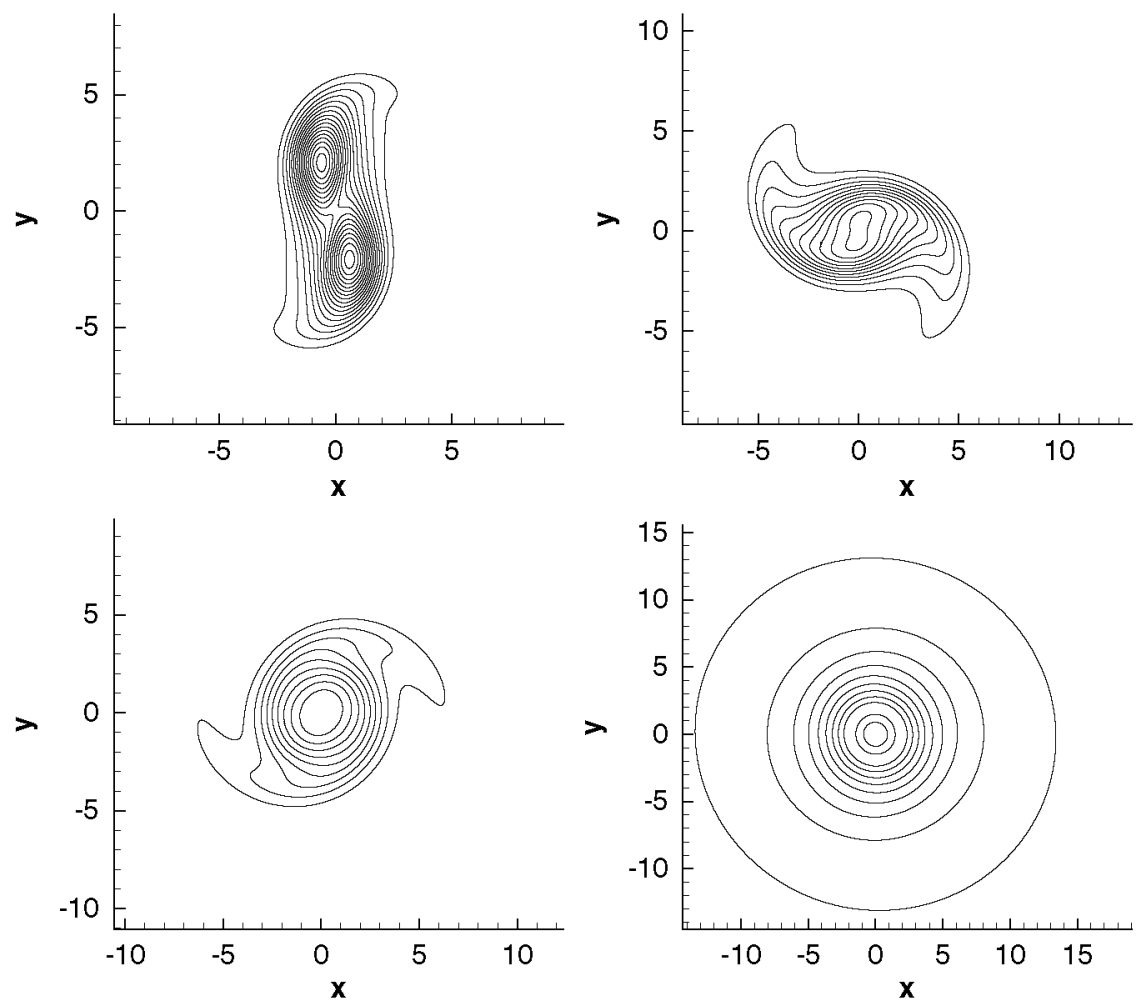

Figure 13. Snapshots of the evolution of the co-rotating vortex pair with $R e=100$ at time: 35 (top left), 70 (top right), 105 (bottom left), 133 (bottom right) seconds obtained by the FEM code $A D F C$ [15].

In the viscous interaction between the two co-rotating vortices three different stages can be identified. The first one is non-viscous and corresponds to a rapid adaptation of each vortex to the external (strain) field generated by the other vortex. This strain field tends to elliptically deform the core of the vortices, see the top left of Figure 13. The second stage consists of a relaxation process dominated by a slow diffusion phenomenon. It is similar to the relaxation of any nonGaussian axisymmetrical vortex towards the Gaussian. The quasi-stationary solution evolves on a viscous-time scale towards a single attractive solution which corresponds to the evolution from two initially Gaussian vortices. In this diffusive stage the vortex radii grow until the separation ratio $a / b$, where $a$ is the effective vortex radius and $b$ is the separation distance between the centres, see the top right and bottom left of Figure 13, gets a critical threshold value $a / b=0.22$ which marks the start of the third stage, the merging process, in which the two vortices merge into a single structure.

In this case study, the viscous temporal evolution of a co-rotating vortex pair of equal circulation $\Gamma$ has been analysed through two different numerical schemes. The WCSPH with the three viscosity models under study and the $A D F C$ finite second-order scheme [15] used to perform the numerical computations of a direct 2D numerical simulation of the incompressible Navier-Stokes equations. The resulting numerical solutions have been compared through the velocity profile at earlier stages of the flow showing good agreement.

In the simulations a circular domain of radius $R$ where the vortex pair evolves has been considered. The initial setting of the particles in $n r$ rings is similar to the one used in previous cases, see Figure 1. The initial condition is the result of the superposition of two Lamb-Ossen vortices centred at $x= \pm 2.5$. The position of the centres of the vortices core fixes the values of the initial separation $b_{0}=5$ and the initial separation rate $a_{0} / b_{0}=0.2$. The Reynolds number for these simulations has been $R e=\Gamma / v=100$, a rather low value that makes it difficult to distinguish the different stages of the vortex evolution described above. 

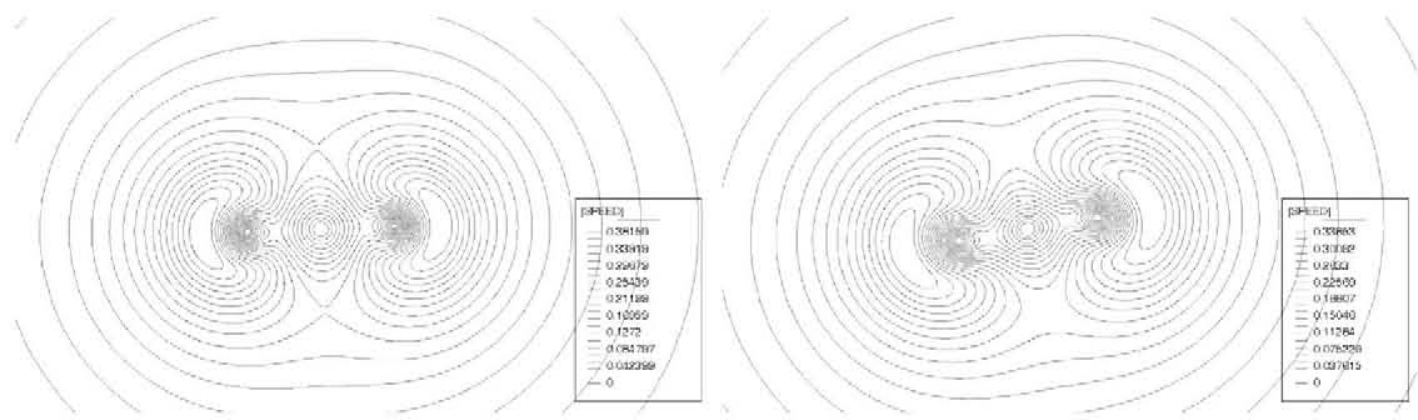

Figure 14. Contour lines of velocity magnitude of the initial evolution of the co-rotating vortex pair at time: 1 (left) and 4 (right) seconds obtained by the FEM code $A D F C$ [15].

For the finite element simulation the number of nodes and Taylor-Hood elements used were 179327 , and 89616 , respectively. The time step was $\Delta t_{s}=0.02 \mathrm{~s}$ and the simulation ran until $T=\Delta t_{s} \cdot N_{t}=0.02 \cdot 350=21 \mathrm{~s}$.

The solution was created by dumping a data file every time unit, see Figure 14 .

The numerical computations for the SPH simulation were repeated many times for different combinations of the following parameters:

- The resolution. Number of rings and number of particles per ring.

- The diameter of the domain.

- The viscosity models (MCGVT, TVT, MVT).

- The initial relaxation time.

- The equations of state.

- The density re-initiation.

The axisymmetry property of the vortex pair for the WCSPH solution is lost when the number of particles in the rows is an odd number, it can be easily restored by forcing an even number of particles per row. Similar to the isolated single vortex case, at the beginning of the experiment all particles are set symmetrically with respect to the origin.

To verify the adequacy of the number of particles in the simulation in order to obtain a solution independent of the number of rings at the fixed Reynolds number, this case study began discussing the independence of the maximum velocity with the increase in the number of particles. Two different number of rings were used $n r=526$ which corresponds to 870591 particles and $n r=590$ with 1095145 particles. Figure 15 shows a stable solution for the MCGVT and the MVT. In contrast, the TVT indicates some differences when the number of particles is increased.

Once we assure that the number of particles was correct, a test to evaluate the influence of the diameter $D$ of the domain in the solution was conducted. The idea is to make most of the runs of the codes with the highest density of particles in the centre of the domain, which is the part of the domain where the most important physics is taking place. This is a common test in the simulation of the vortex interaction of a couple of isolated vortices.

To confirm the irrelevant influence of the no-slip boundary conditions, three different diameters $D=10.0,20.0,30.0$ were used. A noticeable change in the velocity field of the co-rotating vortices for the smallest diameter $D=10$ can be detected in Figure 16. A change that seems to be negligible for diameters larger than 20. Consequently, to eliminate the influence of the diameter, its value has been fixed in the same original value $D=30$ used for the FEM calculation.

To accomplish the main goal of this study, the comparison of the performance of the three selected viscous models was tested with the same conditions, $D=30$ and $n r=526$. Figure 17 shows a similar agreement of the MCGVT and MVT results with the FEM results, and it also manifests some discrepancies between the TVT and FEM results at times $t \sim 6 \mathrm{~s}$. In order to visualize details of the flow when the TVT model presents this inconsistent value, a zoomed snapshot of the particle distribution can be seen in Figure 18. The differences between the results 

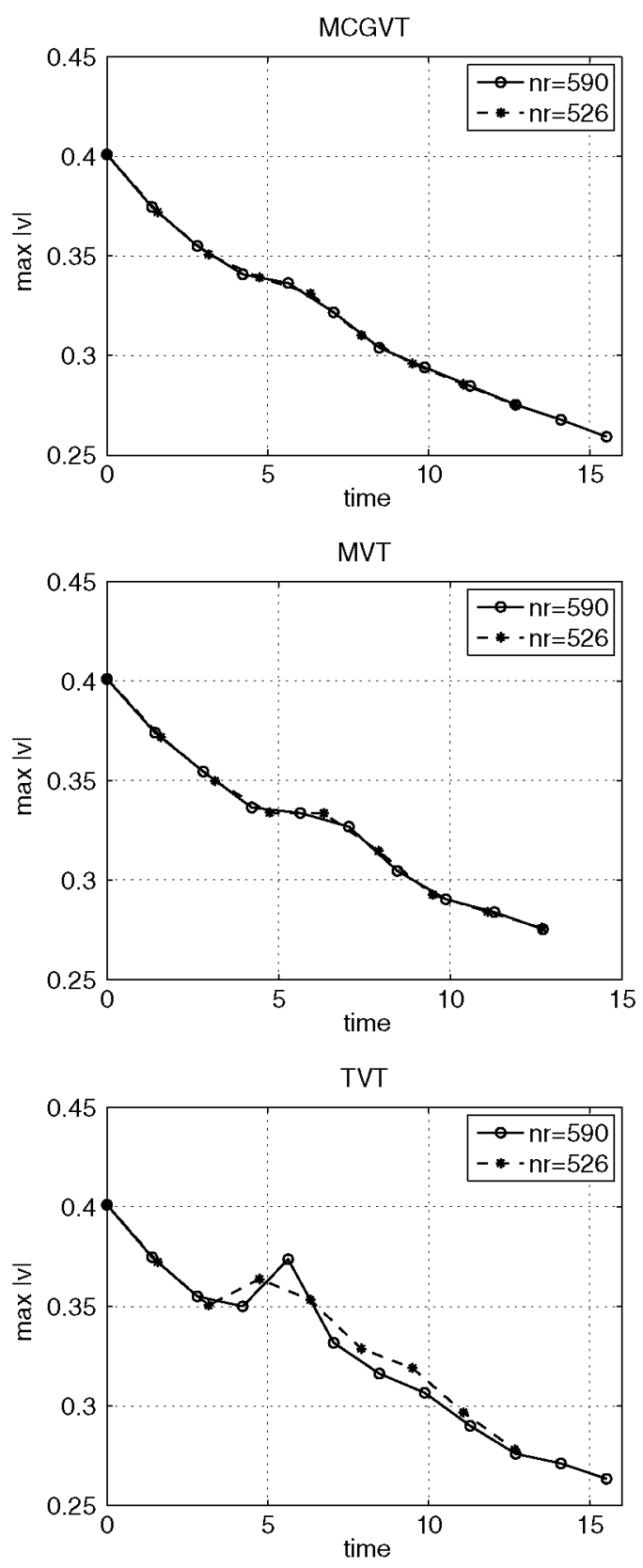

Figure 15. Velocity maximum evolutionary curves for the different viscous models of the co-rotating vortex pair with two different number of rings $n r$. MCGVT (top left), MVT (top right), TVT (bottom).

of the first two SPH viscosity models with the FEM results can be explained by the different approaches to the incompressibility condition. The incompressible assumption of the FEM code versus the weakly compressible hypothesis of the SPH codes. Figure 19 illustrates the comparison at time $t=14 \mathrm{~s}$ of the modulus of the velocity for the three viscosity models with the FEM solution.

Different equations of state have been used in this case to explore any further improvements. Three possibilities were tested, (8) with $\gamma=7$ and $\gamma=1$, and (22). Unfortunately all three possibilities gave similar results with the exception of Equation (22) which performed slightly worse than the others, particularly in the time interval $t \in[3,5]$ seconds, see Figure 20. 


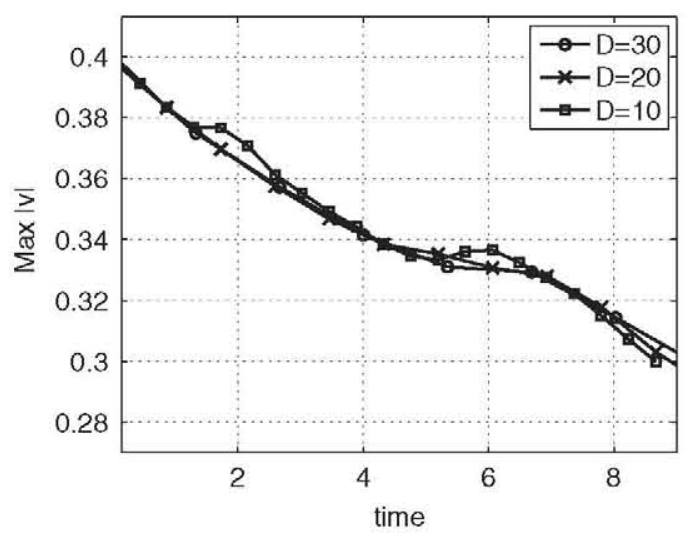

Figure 16. Diameter influence in the evolution of the maximum of the velocity field of the co-rotating vortex pair.

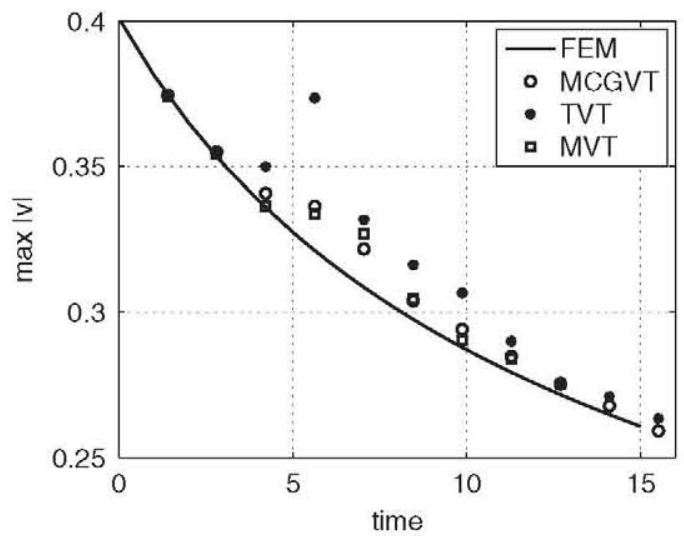

Figure 17. Comparison of the velocity maximum for the different viscous models when $D=30.86$ and $n r=526$.

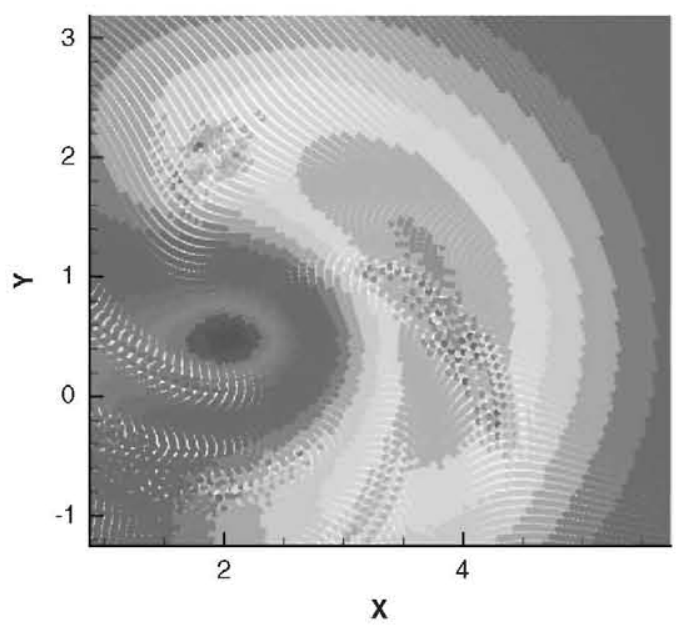

Figure 18. Snapshot of the particle distribution area where the velocity maximum is found at time $t=5.63$ seconds using the TVT. Maximum coordinates $(0.37,4.01)$. 

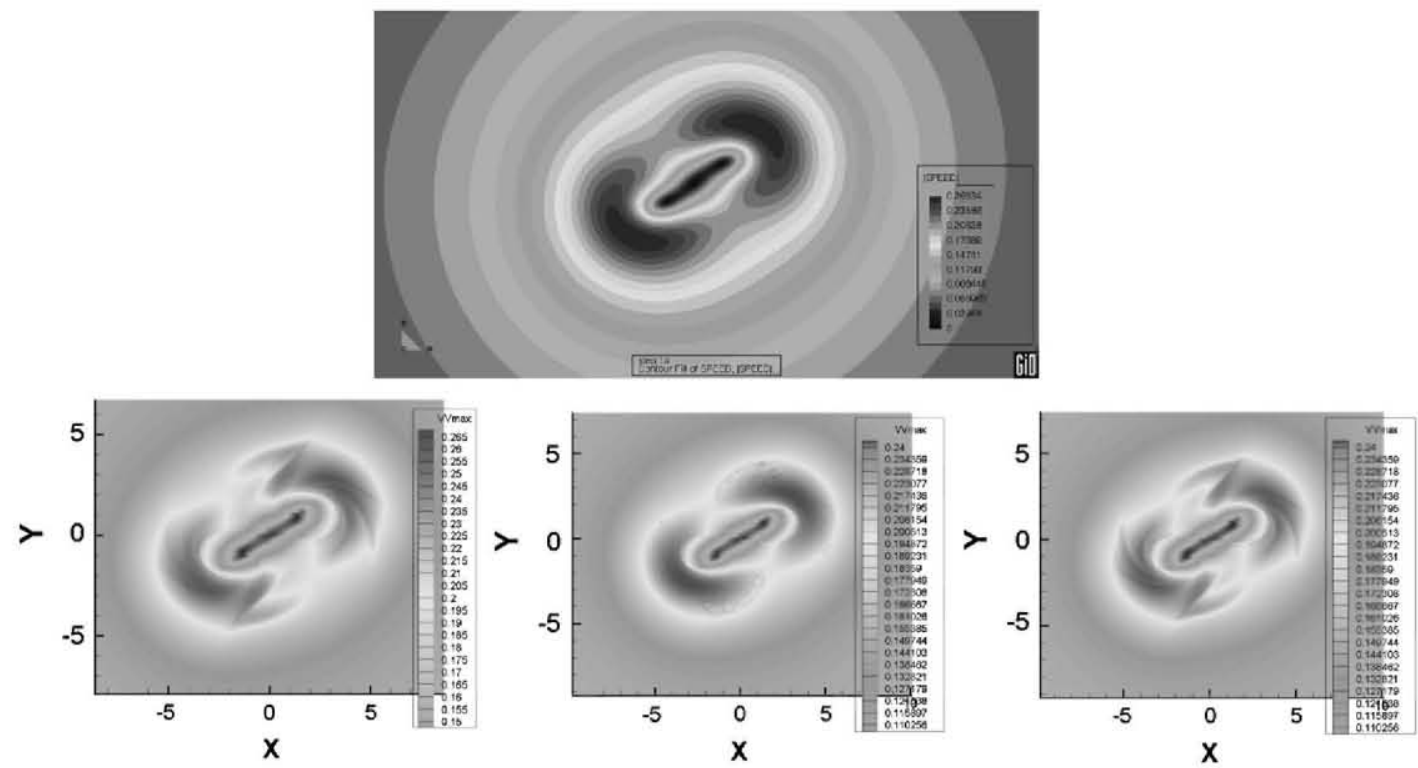

Figure 19. Snapshots of the evolution of the co-rotating couple at time $t=14 \mathrm{~s}$. Top left FEM, top right: SPH (MCGVT), bottom left: SPH (TVT) and bottom right: SPH (MVT).

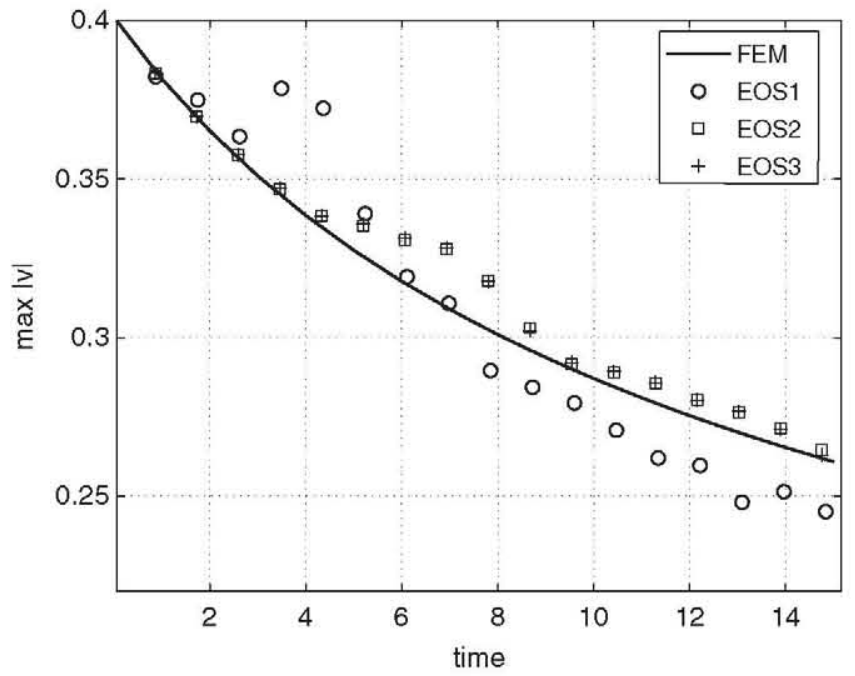

Figure 20. Maximum velocity evolutionary curves for different equations of state. EOS1 corresponds to Equation (22), while EOS2 and EOS3 correspond to Equation (8) with the coefficients $\gamma=7$ and $\gamma=1$, respectively.

To take into account the possible influence of the initial setting of the particles, see [9], the initial setup has been relaxed until the kinetic energy was less than one-tenth of the initial value. There was no evidence of appreciable improvement using this relaxation process.

The last test performed was the examination of the sensitivity with respect to density re-initiation. Following [33], after the predictor-corrector step, the density has been periodically re-initiated using a first-order accurate MLS approximation. This procedure was applied every 5-20 time steps, assuming the inevitable increase in computing time. Negligible changes have been produced when the density has been re-initiated. The insignificant influence of the density re-initiation can be explained in this case in the absence of a free surface or of a second fluid-fluid interface interaction 
in the simulation. In the examples shown in [33] an interface between two fluids is always present and density re-initiation plays an important role.

\section{CONCLUSIONS AND FUTURE WORK}

In this paper, the properties of the most referred SPH laminar viscosity models, Takeda et al. [6], Morris et al. [7] and Monaghan-Cleary-Gingold [8,9], have been explored within the framework of the WCSPH formulation for incompressible flows. A series of test cases have been selected and/or designed aimed at showing the advantages and limitations of these viscous models. Finite element numerical solutions have been derived for both, the Lamb-Oseen vortex evolution and the co-rotating vortex pair flow. For the isolated vortex flow an analytical solution has also been derived. The influence of the boundary conditions, free or no-slip boundary conditions, in the flow has been eliminated. The three models behave similarly for these flows. Their accuracy is good for both a linear-type velocity fields and the more complex one associated with an isolated LambOseen vortex evolution. This conclusion nevertheless does not stand when transport phenomena are relevant in the flow, as in the co-rotating vortex pair evolution, where slight discrepancies in the maximum of the velocity field compared with the reference solution have been found.

Since common Engineering flows embrace both diffusion and transport, it is necessary to clarify the origin and impact of those errors. This may be crucial for the future extension of SPH techniques in the modelling of turbulent flows, transition, small scale dissipation, the simulation of low Reynolds number free surface flows and the modelling of heat diffusion processes.

\section{APPENDIX A: LAMB-OSEEN VORTEX EVOLUTION EXACT SOLUTION}

The vorticity field can be obtained by solving the vorticity-transport equation for incompressible fluids in non-dimensional form, see [34]. The identical formulations in velocity and geometry allows to express the solution in the same frame as the SPH one, by multiplying the time by a factor of $\Gamma=\pi$.

$$
\frac{\partial}{\partial t} \omega(\mathbf{x}, t)=\frac{1}{R e} \nabla^{2} \omega(\mathbf{x}, t) \quad \text { with } \mathbf{x} \in \mathbb{R}^{2}, t \in[0, \infty)
$$

The prescribed initial and boundary conditions are:

$$
\omega(\mathbf{x}, 0)=\omega_{0}(\mathbf{x}), \quad \lim _{|\mathbf{x}| \rightarrow \infty} \omega(\mathbf{x}, t)=0
$$

Let $w$ be the Fourier transform of $\omega$ with respect to $\mathbf{x}$ that is:

$$
w(\chi, t):=\int_{\mathbb{R}^{2}} \omega(\mathbf{x}, t) \mathrm{e}^{-\mathrm{i} \chi \cdot \mathbf{x}} \mathrm{d} \mathbf{x} \quad \text { with } \mathrm{i}:=\sqrt{-1}
$$

A straightforward computation gives

$$
\frac{\partial}{\partial t} w(\chi, t)=-\frac{|\chi|^{2}}{\operatorname{Re}} w(\chi, t)
$$

and therefore,

$$
w(\chi, t)=\mathrm{e}^{-\frac{\mid \chi^{2}}{R e} t} w(\chi, 0)
$$

Applying the Fourier inversion formula, we conclude:

$$
\omega(\mathbf{x}, t)=\frac{R e}{4 \pi t} \int_{\mathbb{R}^{2}} \mathrm{e}^{-\frac{R e}{4 t}|\mathbf{x}-\mathbf{y}|^{2}} \omega_{0}(\mathbf{y}) \mathrm{d} \mathbf{y}
$$


Assuming that $\omega_{0}$ is a LAMB-OSEEN vortex initial datum (27). Formula (A1) gives the following exact expression for the evolution of the LAMB-OSEEN vortex:

$$
\omega(\mathbf{x}, t)=\frac{R e}{4 \pi t} \int_{\mathbb{R}^{2}} \mathrm{e}^{-\left(\frac{R e}{4 t}|\mathbf{x}-\mathbf{y}|^{2}+|\mathbf{y}|^{2}\right)} \mathrm{d} \mathbf{y}
$$

The exact velocity field can be derived from the Biot-Savart law using simple numerical integrations. Its maximum value evolution has been graphically represented and taken as the reference in Figures 9 and 10.

\section{ACKNOWLEDGEMENTS}

The research leading to these results has received funding from the Spanish Ministry for Science and Innovation under grant TRA2010-16988 'Caracterización Numérica y Experimental de las Cargas FluidoDinámicas en el transporte de Gas Licuado'.

\section{REFERENCES}

1. Vila J. On particle weighted methods and smooth particle hydrodynamics. Mathematical Models and Methods in Applied Sciences 1999; 9(2):161-209.

2. Colagrossi A, Delorme L, Colicchio G, Souto-Iglesias A, Cercós-Pita JL. Reynolds number and shallow depth sloshing. Proceedings of the 3rd ERCOFTAC SPHERIC Workshop on SPH Applications, EPFL, Lausanne, Switzerland, 4-6 June 2008; 221-228.

3. Bulian G, Souto-Iglesias A, Delorme L, Botia-Vera E. SPH simulation of a tuned liquid damper with angular motion. Journal of Hydraulic Research, in press.

4. Landrini M, Colagrossi A, Greco M, Tulin MP. Gridless simulations of splashing process and near-shore bore propagation. Journal of Fluid Mechanics 2007; 591:183-213.

5. Chaniotis AK, Poulikakos D, Koumoutsakos P. Remeshed smoothed particle hydrodynamies for the simulation of viscous and heat conducting flows. Journal of Computational Physics 2002; 182:67-90.

6. Takeda H, Miyama SM, Sekiya M. Numerical simulation of viscous flow by smoothed particle hydrodynamics. Progress of Theoretical Physics 1994; 92(5):939-960.

7. Morris JP, Fox PJ, Zhu Y. Modeling low Reynolds number incompressible flows using SPH. Journal of Computational Physics 1997; 136:214-226.

8. Cleary PW. Modelling confined multi-material heat and mass flows using sph. Applied Mathematical Modelling 1998; 22(12):981-993.

9. Monaghan JJ. Smoothed Particle Hydrodynamics simulations of shear flow. Monthly Notices of the Royal Astronomical Society 2005; 365:199-213.

10. Belytschko T, Krongauz Y, Dolbow J, Gerlach C. On the completeness of meshfree particle methods. 1998; 43(5):785-819.

11. Dilts G. Moving-least-squares-particle hydrodynamics-i. Consistency and stability. International Journal for Numerical Methods in Engineering 1999; 44:1115-1155.

12. Dilts G. Moving-least-squares-particle hydrodynamics-ii. Conservation and boundaries. International Journal for Numerical Methods in Engineering 2000; 48:1503-1524.

13. Quinlan NJ, Lastiwka M, Basa M. Truncation error in mesh-free particle methods. International Journal for Numerical Methods in Engineering 2006; 66(13):2064-2085. Available from: http://dx.doi.org/10.1002/nme.1617.

14. Basa M, Quinlan NJ, Lastiwka M. Robustness and accuracy of SPH formulations for viscous flow. International Joumal for Numerical Methods in Fluids 2009; 60(10):1127-1148.

15. González LM, Bermejo R. A semi-Langrangian level set method for incompressible Navier-Stokes equations with free surface. International Journal for Numerical Methods in Fluids 2005; 49:1111-1146.

16. Monaghan JJ. Smoothed particle hydrodynamics. Reports on Progress in Physics 2005; 68:1703-1759.

17. Molteni D, Colagrossi A. A simple procedure to improve the pressure evaluation in hydrodynamic context using the SPH. Computer Physics Communications 2009; 180:861-872.

18. Souto-Iglesias A, Delorme L, Pérez Rojas L, Abril S. Liquid moment amplitude assessment in sloshing type problems with SPH. Ocean Engineering 2006; 33:11-12.

19. Monaghan J. Simulating free surface flows with SPH. Journal of Computational Physics 1994; 110(2):399-406.

20. Lee ES, Moulinec C, Xu R, Violeau D, Laurence D, Stansby P. Comparisons of weakly compressible and truly incompressible algorithms for the SPH mesh free particle method. Joumal of Computational Physics 2008; 227(18):8417-8436.

21. Monaghan J, Gingold R. Shock simulation by the particle method SPH. Journal of Computational Physics 1983; 52:374-389.

22. Cleary PW, Monaghan JJ. Boundary interactions and transition to turbulence for standard CFD problems using SPH. Proceeding of 6 th Intemational Computational Techniques and Applications Conference, Canberra, 1993; 157-165. 
23. Hu X, Adams NA. Angular-momentum conservative smoothed particle hydrodynamics for incompressible viscous flows. Physics of Fluids 2006; 18:702-706.

24. Brookshaw L. A method of calculating radiative heat diffusion in particle simulations. Proceedings of the Astronomical Society of Australia 1985; 6(2):207-210.

25. Español P, Revenga M. Smoothed dissipative particle dynamics. Physical Review E 2003; 67:026705 1-12.

26. Cleary P. New implementation of viscosity. Tests with Couette flows. CSIRO Division of Mathematics and Statistics, Technical Reports, May 1996.

27. Wagner A, Li Q. Investigation of galilean invariance of multi-phase Lattice Boltzmann methods. Physica A: Statistical Mechanics and its Applications 2006; 362(1):105-110, discrete Simulation of fluid dynamicsProceedings of the 13th International Conference on Discrete Simulation of Fluid Dynamics, The 13th International Conference on Discrete Simulation of Fluid Dynamics. Available from: http://www.sciencedirect.com/science/ article/B6TVG-4H88312-3/2/c74a1435f1787527cde8720dfea8da6b.

28. Batchelor GK. Introduction to Fluid Dynamics. Cambridge University Press: New York, 1967.

29. Monaghan J. SPH without a tensile instability. Journal of Computational Physics 2000; 159(2):290-311.

30. Imaeda Y, Inutsuka S. Shear flows in smoothed particle hydrodynamics. Astrophysical Journal 2002; 569(1):501-518.

31. Souto-Iglesias A, González LM, Colagrossi A, Antuono M. SPH no-slip BC implementation analysis at the continuous level. Proceedings of the 5th ERCOFTAC SPHERIC Workshop on SPH Applications, University of Manchester, U.K., 23-25 June 2010; 29-36.

32. Le Dizès S, Verga A. Viscous interactions of two co-rotating vortices before merging. Journal of Fluid Mechanics 2002; 467:389-410.

33. Colagrossi A, Landrini M. Numerical simulation of interfacial flows by smoothed particle hydrodynamics. Journal of Computational Physics 2003; 191:448-475.

34. Majda AJ, Bertozzi AL. Vorticity and Incompressible Flow, Series in Cambridge Texts in Applied Mathematics, vol. 27. Cambridge University Press: Cambridge, 2002. 